\title{
Replicating the properties of hedge fund returns *
}

\author{
Nicolas Papageorgiou, Bruno Rémillard, Alexandre Hocquard \\ HEC Montréal
}

April 8, 2008

\begin{abstract}
In this paper, we implement a multi-variate extension of Dybvig (1988) Payoff Distribution Model that can be used to replicate not only the marginal distribution of most hedge fund returns but also their dependence with other asset classes. In addition to proposing ways to overcome the hedging and compatibility inconsistencies in Kat and Palaro (2005), we extend the results of Schweizer (1995) and adapt American options pricing techniques to evaluate the model and also derive an optimal dynamic trading (hedging) strategy. The proposed methodology can be used as a benchmark for evaluating fund performance, as well as to replicate hedge funds or generate synthetic funds.
\end{abstract}

Key Words: Hedge Funds, Hedging, Replication, Copula, Gaussian mixtures.

J.E.L. classification: G10, G20, G28, C16

\footnotetext{
${ }^{*}$ Corresponding author: Nicolas Papageorgiou, Finance Department, HEC Montréal, 3000 Cote SainteCatherine, Montreal,QC, H3T 2A7, Canada. All the authors are at HEC Montréal and can be reached at firstname.lastname@hec.ca. This work is supported in part by the Fonds pour la formation de chercheurs et l'aide à la recherche du Gouvernement du Québec, by the Fonds québécois de la recherche sur la société et la culture, by the Natural Sciences and Engineering Research Council of Canada and by Desjardins Global Asset Management. The authors would also like to thank Frank Leclerc, Aziz Soré, Gauthier Webanck and Hugues Langlois.
} 


\section{Introduction}

The impressive growth of the hedge fund industry has naturally led to an increased scrutiny of the fund managers and of their investment strategies. Given the often exorbitant management and performance fees charged by hedge fund managers, it is not surprising that investors are starting to question what they are actually getting for their money. Shrewd investors and institutional fund of funds are becoming increasingly careful about paying alpha fees for beta returns. The challenge that investors and researchers are therefore confronted with is how to reliably separate the funds that are generating alpha returns from the ones that are simply repackaging beta.

The approach that has generally been favored by academics and practitioners in order to extract information about hedge fund returns is the factor model approach. The underlying idea is to try and separate the returns that are due to systematic exposure to risk factors (beta returns) from those that are due to managerial skill (alpha returns). Once the relevant risk factors have been identified, one can evaluate whether the funds exhibit abnormal returns based the intercept of a linear regression of the fund returns against the factor returns. A further advantage of this methodology is that if the linear model is wellspecified, one can attempt to replicate the returns of the hedge fund by investing in the appropriate portfolio of factors. A recent paper by Hasanhodzic and Lo (2007) provides some evidence that linear replication can be successful for certain strategies whilst offering certain advantages to hedge fund investing. These include more transparency, increased liquidity and fewer capacity constraints. However the authors warn that the heterogeneous risk profile of hedge funds and the non-linear risk exposures greatly reduce the ability of these models to consistently replicate hedge fund returns. Over the last few months, several banks including Goldman Sachs, JP Morgan and Merril Lynch have launched linear replication funds.

Certain generic hedge fund characteristics help explain some of the difficulty in identifying a well specified linear model. The use of financial derivatives, the use of dynamic leverage, the use of dynamic trading strategies and the asymmetric performance fee structures are some of the most obvious sources of non-linearities in hedge fund returns. Several recent papers, such as Mitchell and Pulvino (2001), Fung and Hsieh (2001), Agarwal and Naik (2004), Chen and Liang (2006), Kazemi and Schneeweis (2003) have dealt with the inclusion of risk premia and conditional betas that attempt to account for these non-linearities. The inclusion of the above option-based factors significantly improves the explanatory power of factor models, however, most of these factors are not tradable and therefore cannot be used to construct a replicating portfolio.

In order to circumvent the issue of identifying tradable risk factors, an interesting alternative approach was proposed by Amin and Kat (2003) and more recently extended by Kat and Palaro (2005). Based on earlier work by Dybvig (1988), the authors evaluate hedge 
fund performance not by identifying the return generating betas, but rather by attempting to replicate the distribution of the hedge fund returns. The underlying idea is based on the hypothesis that much of the trading activity undertaken by hedge funds is not creating value, just altering the timing of the returns available from traditional assets. In effect, many hedge funds are simply distorting readily available asset distributions. So the real challenge is whether or not we can find a more efficient method to distort these distributions than by investing in hedge funds. Armed with their new efficiency measure, Kat and Palaro (2005) show that hedge fund returns are by no means exceptional and that for the majority of funds an alternative dynamic strategy would have provided investors with superior returns. This methodology not only provides a model free benchmark for evaluating hedge funds, it can also be used to create synthetic funds with predetermined distributional properties.

The efficiency measure as presented by Kat and Palaro (2005) is however subject to several shortcomings and inconsistencies. The most significant of these relates to the way that the daily trading strategies are derived from the distribution of monthly returns. The properties of the estimated monthly distributions and copula functions proposed by the authors are not infinitely divisible and therefore the true properties of the daily returns are not known. As a result, the replicating strategy will not be precise. A further weakness pertains to the fact that although the hedge fund returns and traded assets are clearly nonnormal, the efficiency measure is calculated within the confines of the Black-Scholes-Merton world, hence ignoring the higher moments of the distributions.

In this paper, we will implement a multi-variate extension of Dybvig (1988) Payoff Distribution Model that can be used to replicate not only the marginal distribution of most hedge fund returns but also their dependence with other asset classes. In addition to proposing ways to overcome the hedging and compatibility inconsistencies in previous papers, we extend the results of Schweizer (1995) and adapt American options pricing techniques to evaluate the model and also derive an optimal dynamic trading (hedging) strategy. The proposed methodology can be used as a benchmark for evaluating fund performance, as well as to replicate hedge funds or generate synthetic funds.

The rest of the paper will be structured as follows. Section 2 will explain the intuition behind our multi-variate extension of Dybvig's Payoff Distribution model. Section 3 presents the technical details relating to the modeling and estimation of the distributions. Section 4 presents the payoff function. Section 5 presents the replication issues and presents the optimal dynamic trading strategy. Section 6 presents some numerical results. Section 7 concludes. 


\section{The Multivariate Payoff Distribution Model}

In Kat and Palaro (2005), the authors show that given two risky assets $S^{(1)}$ and $S^{(2)}$, it is possible to "reproduce" the statistical properties of the joint return distribution of asset $S^{(1)}$ and a third asset $S^{(3)}$. Let's assume asset $S^{(1)}$ is the investor portfolio, asset $S^{(2)}$ is a tradable security and asset $S^{(3)}$ is a hedge fund, this result implies that we can generate the distribution of the hedge fund and its dependence with the investor portfolio, by only investing in the tradable security $S^{(2)}$ and the investor portfolio $S^{(1)}$. Note that we do not replicate the month by month returns of the hedge fund, but instead we replicate its distributional properties (i.e. expectation, volatility, skewness and kurtosis) as well as dependence measures with respect to the returns of the investor portfolio (i.e. Pearson, Spearman correlations...).

Essentially, there exist a payoff function that will allow us to transform the joint distribution of assets $S^{(1)}$ and $S^{(2)}$ into the bivariate distributions of $S^{(1)}$ and $S^{(3)}$. This payoff function is easily shown to be calculable using the marginal distribution functions $F_{1}$, $F_{2}$ and $F_{3}$ of $S_{T}^{(1)}, S_{T}^{(2)}, S_{T}^{(3)}$, and the copulas $\mathcal{C}_{1,2}$ and $\mathcal{C}_{1,3}$ associated respectively with the

joints returns $\left(R_{0, T}^{(1)}, R_{0, T}^{(2)}\right)$ and $\left(R_{0, T}^{(1)}, R_{0, T}^{(3)}\right)$. The exact expression for the payoff function is given in section 4 .

The challenge that we are confronted with is how to best evaluate this function, and this is by no means a trivial problem. The problem can however be broken down into three separate components. The first part relates to the proper modeling of the distributions and copula functions. The second part consists in calculating the payoff function. The third part consists in selecting an approach that will allow us to generate a dynamic trading strategy that provides us with the best possible approximation of the payoff function.

\section{Modeling the returns}

In order to provide a robust solution in this framework, we propose the following steps. First, we will model the joint daily distribution of $S^{(1)}$ and $S^{(2)}$ using bivariate Gaussian mixtures. Since we will be trading these assets on a daily basis, it is imperative that the distribution of the monthly returns for both the investor portfolio and the reserve asset are consistent with the distribution of the daily returns. We need to be sure that by dynamically trading the assets based on the joint daily distributions we will be able to generate the desired monthly properties. We will therefore estimate the parameters of the bivariate Gaussian mixtures of $R_{t}$, (investor portfolio and reserve asset) using the historical daily returns of $S^{(1)}$ and $S^{(2)}$. We can then solve for the law of the monthly returns that is compatible with the law of daily returns. Furthermore, the daily dependence which is modeled with the bivariate mixtures will allow us to obtain the desired monthly dependence. This would not have been possible if we used univariate laws to model the marginal distributions and a copula to model the 
dependence structure. Although copula provide us with much flexibility in terms of modeling the dependence, there is however no proof to this day that the statistical properties of copula functions are divisible. Finally, we need to estimate the monthly distribution of the hedge fund returns as well as the dependence between the hedge fund and the investor portfolio. There are no particular restrictions regarding the choice of the distribution of $S^{(3)}$ and the copula $\mathcal{C}_{1,3}$. We have developed statistical tests that allow us to select the most appropriate marginal distribution and copula function. We now consider each of these steps in detail.

\subsection{Mixtures of Gaussian distributions}

The choice of Gaussian mixtures to model the bivariate distribution of investor portfolio and the reserve asset is due to both the flexibility of the mixtures in capturing high levels of skewness as well as the fact that the bivariate distribution is infinitely divisible. In this section, we will first provide a brief description of bivariate Gaussian mixtures and discuss their statistical properties. Finally we will present a goodness-of-fit test that we developed in order to estimate the mixtures and select the optimal number of regimes.

\subsubsection{Definition of mixtures of Gaussian bivariate vectors}

A bivariate random vector $X$ is a Gaussian mixture with $m$ regimes and parameters $\left(\pi_{k}\right)_{k=1}^{m}$, $\left(\mu_{k}\right)_{k=1}^{m}$ and $\left(A_{k}\right)_{k=1}^{m}$, if its density is given by

$$
f(x)=\sum_{k=1}^{m} \pi_{k} \phi_{2}\left(x ; \mu_{k}, A_{k}\right)
$$

where $\phi_{2}(x ; \mu, A)=\frac{e^{-\frac{1}{2}(x-\mu)^{\top} A^{-1}(x-\mu)}}{2 \pi \sigma_{1} \sigma_{2}\left(1-\rho^{2}\right)^{1 / 2}}$ is the density of a bivariate Gaussian vector with mean vector $\mu=\left(\mu_{1}, \mu_{2}\right)^{\top}$ and covariance matrix $A=\left(\begin{array}{cc}\sigma_{1}^{2} & \rho \sigma_{1} \sigma_{2} \\ \rho \sigma_{1} \sigma_{2} & \sigma_{2}^{2}\end{array}\right)$. Its distribution function is

$$
F\left(x_{1}, x_{2}\right)=\sum_{k=1}^{m} \pi_{k} \Phi_{2}\left(\frac{x_{1}-\mu_{k 1}}{\sigma_{k 1}}, \frac{x_{2}-\mu_{k 2}}{\sigma_{k 2}} ; \rho_{k}\right),
$$

where $\Phi_{2}(\cdot, \cdot ; \rho)$ is the bivariate standard Gaussian distribution function with correlation $\rho$.

\subsubsection{Some properties of mixtures of bivariate Gaussian variables}

One property that is quite important in our setting is the fact that a sum of independent Gaussian mixtures is still a Gaussian mixture. In fact, if $X_{1}, \ldots, X_{n}$ are independent and 
identically Gaussian mixtures with parameter $\theta$, then $X=X_{1}+\cdots+X_{n}$ is also a Gaussian mixture. To describe the associated parameters, let

$$
\mathcal{A}=\left\{\alpha=\left(\alpha_{1}, \ldots, \alpha_{m}\right) ; \alpha_{j} \geq 0 \text { and } \alpha_{1}+\ldots+\alpha_{m}=n\right\} .
$$

Then $\operatorname{card}(\mathcal{A})=\left(\begin{array}{c}n+m-1 \\ m-1\end{array}\right)$ so there are $\left(\begin{array}{c}n+m-1 \\ m-1\end{array}\right)$ regimes. The parameters of the mixture are $\left(\pi_{\alpha}\right)_{\alpha \in \mathcal{A}},\left(\mu_{\alpha}\right)_{\alpha \in \mathcal{A}},\left(A_{\alpha}\right)_{\alpha \in \mathcal{A}}$, where for each $\alpha \in \mathcal{A}, \pi_{\alpha}$ is the multinomial probability

$$
\pi_{\alpha}=\pi_{\left(\alpha_{1}, \cdots, \alpha_{m}\right)}=\frac{n !}{\alpha_{1} ! \cdots \alpha_{m} !} \prod_{k=1}^{m} \pi_{k}^{\alpha_{k}},
$$

and the mean vectors $\mu_{\alpha}$ and covariances $A_{\alpha}$ are respectively given by

$$
\mu_{\alpha}=\sum_{k=1}^{n} \alpha_{k} \mu_{k}, \quad A_{\alpha}=\sum_{k=1}^{n} \alpha_{k} A_{k} .
$$

Remark 3.1 If $n$ is moderately large, then $m^{n}$ is huge and it is computationally impossible to calculate the new parameters. In fact, most probabilities could be very small so in fact, the sum could be a mixture of fewer terms. Therefore, one has to estimate again the joint law of $\left(R_{0, T}^{(1)}, R_{0, T}^{(2)}\right)$ by a Gaussian mixture, using the monthly returns this time. As a result, the marginal distributions $F_{1}$ and $F_{2}$ are (univariate) Gaussian mixtures and $\mathcal{C}_{1,2}$ is the copula deduced from the bivariate Gaussian mixture.

Finally, consider the conditional distribution of a bivariate Gaussian mixture $X=$ $\left(X^{(1)}, X^{(2)}\right)$. Set $\beta_{k}=\rho_{k} \frac{\sigma_{k 2}}{\sigma_{k 1}}$ and $\alpha_{k}=\mu_{k 2}-\beta_{k} \mu_{k 1}, k=1, \ldots, m$. Then it is easy to check that the conditional distribution of $X^{(2)}$ given $X^{(1)}=x_{1}$ is a Gaussian mixture with parameters $\left\{\tilde{\pi}_{k}\left(x_{1}\right)\right\}_{k=1}^{m},\left\{\tilde{\mu}_{k}\left(x_{1}\right)\right\}_{k=1}^{m},\left\{\tilde{\sigma}_{k}^{2}\right\}_{k=1}^{m}$, where

$$
\tilde{\pi}_{k}\left(x_{1}\right)=\frac{\pi_{k} \phi\left(x_{1} ; \mu_{k 1}, \sigma_{k 1}^{2}\right)}{\sum_{j=1}^{m} \pi_{j} \phi\left(x_{1} ; \mu_{j 1}, \sigma_{j 1}^{2}\right)}
$$

and

$$
\tilde{\mu}_{k}\left(x_{1}\right)=\alpha_{k}+\beta_{k} x_{1}, \quad \tilde{\sigma}_{k}^{2}=\sigma_{k}^{2}\left(1-\rho_{k}^{2}\right) .
$$

\subsubsection{Estimation and goodness-of-fit}

In order to choose the optimal number of regimes, we need to first estimate the parameters of the model, and then provide a goodness-of-fit test to evaluate whether a greater number of regimes is required. The estimation method is based on the EM algorithm of (Dempster et al., 1977). It is presented in Appendix A.1 for the bivariate case. 
A new goodness-of-fit test, described in Appendix A.4, can be performed to assess the suitability as well as to select the number of mixture regimes $m$. The proposed test, based on the work in Genest et al. (2007), uses the Rosenblatt's transform.

For the selection of the number $m$ of regimes, the following two steps procedure is suggested:

(a) Find the first $m_{0}$ for which the $P$-value of the tests described in A.4 is larger than $5 \%$.

(b) Estimate parameters for $m_{0}+1$ regimes and apply the likelihood ratio test to check if the null hypothesis $H_{0}: m=m_{0}$ vs $H_{1}: m=m_{0}+1$. If $H_{0}$ is rejected at the $5 \%$ level, repeat steps (a) and (b) starting at $m=m_{0}+1$. However, if the parameters under $H_{1}$ yield a degenerate density (e.g., $\left|\rho_{k}\right|=1$ ), stop and set $m=m_{0}$.

\subsection{Choice/estimation of the marginal distribution $F_{3}$}

There are no restrictions on the choice of $F_{3}$, which is the distribution of the hedge fund that we seek to replicate (or the desired distribution in the case of a synthetic fund). Unlike the reserve asset and investor portfolio that require divisible laws, we are only interested in monthly return distribution and hence can introduce any distribution. In the case of the replication of an existing hedge fund, goodness-of-fit is important and therefore we test using a Durbin type test, as described in Appendix A.3.

\subsection{Choice/estimation of the copula $\mathcal{C}_{1,3}$}

Again, there are no restrictions on the choice of copula function $\mathcal{C}_{1,3}$, between the monthly returns of the hedge fund and the investor portfolio. Suppose that we have historical monthly returns $\left(Y_{1}, Z_{1}\right), \ldots,\left(Y_{n}, Z_{n}\right)$ belong to a copula family $C_{\theta}$. To estimate $\theta$, one often uses the so-called IFM method. However, we do not recommend it as the parameters of the copula function rely on the estimated marginal distributions. Any mis-specification of the marginal distributions will bias the choice of copula. For reasons of robustness, it is therefore preferable to use normalized ranks, i.e. if $R_{i 1}$ represents the rank of $Y_{i}$ among $Y_{1}, \ldots, Y_{n}$ and if $R_{i 2}$ represents the rank of $Z_{i}$ among $Z_{1}, \ldots, Z_{n}$, with $R_{i j}=1$ for the smallest observations, then set

$$
U_{i}=\frac{R_{i 1}}{n+1}, \quad V_{i}=\frac{R_{i 2}}{n+1}, \quad i=1, \ldots, n .
$$

To estimate $\theta$ one could try to maximize the pseudo-log-likelihood

$$
\sum_{i=1} \log c_{\theta}\left(U_{i}, V_{i}\right)
$$


as suggested in Genest et al. (1995). For example, if the copula is the Gaussian copula with correlation $\rho$, the pseudo-likelihood estimator for $\rho$ yields the famous van der Waerden coefficient defined to be the correlation between the pairs $\left\{\Phi^{-1}\left(U_{i}\right), \Phi^{-1}\left(V_{i}\right) ; i=1, \ldots, n\right\}$. For other families that can be indexed by Kentall's tau, e.g., Clayton, Frank and Gumbel families, one could estimate the parameter by inversion of the sample Kendall's tau. See, e.g., Genest et al. (2006).

Finally, to test for goodness-of-fit, one can use Cramér-von Mises type statistics for the empirical copula or for the Rosenblatt's transform. The latter could be the best choice given that $\frac{\partial}{\partial u} \mathcal{C}_{1,3}(u, v)$ needed to be calculated for the evaluation of the payoff function. These tests are described in Genest et al. (2007) and in view of their results, we recommend to use the test statistic $S_{n}^{(B)}$.

\section{The payoff function}

Having estimated the necessary distributions and copula function, one must now calculate the payoff's return function $g$. As deduced by Kat and Palaro (2005), its formula is given by

$$
g(x, y)=Q\left\{x, P\left(R_{0, T}^{(2)} \leq y \mid R_{0, T}^{(1)}=x\right)\right\}
$$

where $Q(x, \alpha)$ is the order $\alpha$ quantile of the conditional law of $R_{0, T}^{(3)}$ given $R_{0, T}^{(1)}=x$, i.e., for any $\alpha \in(0,1), q(x, \alpha)$ satisfies

$$
P\left\{R_{0, T}^{(3)} \leq Q(x, \alpha) \mid R_{0, T}^{(1)}=x\right\}=\alpha .
$$

Using properties of copulas, e.g. Nelsen (1999), the conditional distributions can be expressed in terms of the margins and the associated copulas.

$$
P\left(R_{0, T}^{(2)} \leq y \mid R_{0, T}^{(1)}=x\right)=\left.\frac{\partial}{\partial u} \mathcal{C}_{1,2}(u, v)\right|_{u=F_{1}(x), v=F_{2}(y)} .
$$

Note that $\frac{\partial}{\partial u} \mathcal{C}_{1,2}(u, v)=P\left\{F_{2}\left(R_{0, T}^{(2)}\right) \leq v \mid F_{1}\left(R_{0, T}^{(1)}\right)=u\right\}$. In addition, if $\mathcal{Q}(u, \alpha)$ is the order $\alpha$ quantile of the distribution function $\frac{\partial}{\partial u} \mathcal{C}_{1,3}(u, v)$, then one obtains

$$
Q(x, \alpha)=F_{2}^{-1} \circ \mathcal{Q}\left(F_{1}(x), \alpha\right) .
$$

In our methodology, since the monthly returns $\left(R_{0, T}^{(1)}, R_{0, T}^{(2)}\right)$ are modeled by a Gaussian mixtures with parameters $\left(\pi_{k}\right)_{k=1}^{m},\left(\mu_{k}\right)_{k=1}^{m}$ and $\left(A_{k}\right)_{k=1}^{m}$, the conditional distributions 
can be expressed as follows

$$
P\left(R_{0, T}^{(2)} \leq y \mid R_{0, T}^{(1)}=x\right)=\sum_{k=1}^{m} \tilde{\pi}_{k}(x) \phi\left\{y ; \tilde{\mu}_{k}(x), \tilde{\sigma}^{2}\right\}
$$

where $\tilde{\pi}_{k}(x), \tilde{\mu}_{k}(x)$ and $\tilde{\sigma}^{2}$ are given by formulas (1) and (2).

\section{Dynamic replication}

Having solved for $g$, we need to find an optimal dynamic trading strategy that will replicate the payoff function. We do so by selecting the portfolio $\left(V_{0}, \varphi\right)$ such as to minimize the expected square hedging error

$$
E\left[\beta_{T}^{2}\left\{V_{T}\left(V_{0}, \varphi\right)-C_{T}\right\}^{2}\right]
$$

where $\beta_{T}$ is the discount factor and $C_{T}=100 \exp \left\{g\left(R_{0, T}^{(1)}, R_{0, T}^{(2)}\right)\right\}$ is the payoff at maturity.

In order to achieve this, we develop extensions of the results of Schweizer (1995). Note that there is no "risk-neutral" evaluation involved in our approach and that all calculations are carried out under the objective probability measure.

If the dynamic replication is successful, i.e., $V_{T}=C_{T}$, then return of the investment can be decomposed as

$$
\log \left(V_{T} / V_{0}\right)=\log \left(100 / V_{0}\right)+g\left(R_{0, T}^{(1)}, R_{0, T}^{(2)}\right) .
$$

Therefore, as proposed in Kat and Palaro (2005), one can view $\alpha=\log \left(100 / V_{0}\right)$ as a measure of performance. For, if $\alpha=0$, we generate exactly the target distribution, while if $\alpha>0$, we outperform the target distribution; if $\alpha<0$, then the fund outperforms the replication strategy. However, whatever the value of $\alpha$, statistics based on centered moments are not affected; only the value of the expectation depends on $\alpha$.

\subsection{Optimal hedging}

Suppose that $(\Omega, P, \mathcal{F})$ is a probability space with filtration $\mathbb{F}=\left\{\mathcal{F}_{0}, \ldots, \mathcal{F}_{T}\right\}$, under which the stochastic processes are defined. For the moment, assume that the price process $S_{t}$ is $d$-dimensional, i.e. $S_{t}=\left(S_{t}^{(1)}, \ldots, S_{t}^{(d)}\right)$. In the next section, one will come back with the case $d=2$. 
Before defining what is meant by a dynamic replicating strategy, let $\beta_{t}$ denote the discount factor, i.e. $\beta_{t}$ is the value at period 0 to be invested in the non risky asset so that it has a value of $1 \$$ at period $t$. By definition, $\beta_{0}=1$. It is assumed that the process $\beta$ is predictable, i.e. $\beta_{t}$ is $\mathcal{F}_{t-1}$-measurable for all $t=1, \ldots, T$.

A dynamic replicating strategy can be described by a (deterministic) initial value $V_{0}$ and a sequence of random weight vectors $\varphi=\left(\varphi_{t}\right)_{t=0}^{T}$, where for any $j=1, \ldots, d, \varphi_{t}^{(j)}$ denotes the number of parts of assets $S^{(j)}$ invested during period $(t-1, t]$. Because $\varphi_{t}$ may depend only on the values values $S_{0}, \ldots, S_{t-1}$, the stochastic process $\varphi_{t}$ is assumed to be predictable. Initially, $\varphi_{0}=\varphi_{1}$, and the portfolio initial value is $V_{0}$. It follows that the amount initially invested in the non risky asset is

$$
V_{0}-\sum_{j=1}^{d} \varphi_{1}^{(j)} S_{0}^{(j)}=V_{0}-\varphi_{1}^{\top} S_{0}
$$

Since the hedging strategy must be self-financing, it follows that for all $t=1, \ldots, T$,

$$
\beta_{t} V_{t}\left(V_{0}, \varphi\right)-\beta_{t-1} V_{t-1}\left(V_{0}, \varphi\right)=\varphi_{t}^{\top}\left(\beta_{t} S_{t}-\beta_{t-1} S_{t-1}\right)
$$

Using the self-financing condition (3), it follows that

$$
\beta_{T} V_{T}=\beta_{T} V_{T}\left(V_{0}, \varphi\right)=V_{0}+\sum_{t=1}^{T} \varphi_{t}^{\top}\left(\beta_{t} S_{t}-\beta_{t-1} S_{t-1}\right)
$$

The replication strategy problem for a given payoff $C$ is thus equivalent to finding the strategy $\left(V_{0}, \varphi\right)$ so that the hedging error

$$
G_{T}\left(V_{0}, \varphi\right)=\beta_{T} V_{T}\left(V_{0}, \varphi\right)-\beta_{T} C
$$

is as small as possible. In this paper, we choose the expected square hedging error as a measure of quality of replication. It is therefore natural to suppose that the prices $S_{t}^{(j)}$ have finite second moments. We further assume that the hedging strategy $\varphi$ satisfies a similar property, namely that for any $t=1, \ldots, T, \varphi_{t}^{\top}\left(\beta_{t} S_{t}-\beta_{t-1} S_{t-1}\right)$ have finite second moments. Note that these two technical conditions were also made by Schweizer (1995).

For simplicity, set

$$
\Delta_{t}=S_{t}-E\left(S_{t} \mid \mathcal{F}_{t-1}\right), \quad t=1, \ldots, T
$$

Under the above moment conditions, the conditional covariance matrix $\Sigma_{t}$ of $\Delta_{t}$ exists and is given by

$$
\Sigma_{t}=E\left\{\Delta_{t} \Delta_{t}^{\top} \mid \mathcal{F}_{t-1}\right\}, 1 \leq t \leq T
$$


In Schweizer (1995), the author treats the case $d=1$ and assumes a restrictive boundedness condition. Here, in contrast, we treat the general $d$-dimensional case and we only suppose that $\Sigma_{t}$ is invertible for all $t=1, \ldots, T$. This was implicitly part of the boundedness condition of Schweizer (1995).

If $\Sigma_{t}$ is not invertible for some $t$, there would exists a $\varphi_{t} \in \mathcal{F}_{t-1}$ such that $\varphi_{t}^{\top} S_{t}=$ $\varphi_{t}^{\top} E\left(S_{t} \mid \mathcal{F}_{t-1}\right)$, that is, $\varphi_{t}^{\top} S_{t}$ is predictable. Our assumption can be interpreted as saying that the genuine dimension of the assets is $d$. One may now state the main result whose proof is given in Appendix D.1.

Theorem 1 Suppose that $\Sigma_{t}$ is invertible for all $t=1, \ldots, T$. Then the risk $E\left\{G^{2}\left(V_{0}, \varphi\right)\right\}$ is minimized by choosing recursively $\varphi_{T}, \ldots, \varphi_{1}$ satisfying

$$
\varphi_{t}=\left(\Sigma_{t}\right)^{-1} E\left(\left\{S_{t}-E\left(S_{t} \mid \mathcal{F}_{t-1}\right)\right\} C_{t} \mid \mathcal{F}_{t-1}\right), t=T, \ldots, 1,
$$

where $C_{T}, \ldots, C_{0}$ are defined recursively by setting $C_{T}=C$ and

$$
\beta_{t-1} C_{t-1}=\beta_{t} E\left(C_{t} \mid \mathcal{F}_{t-1}\right)-\varphi_{t}^{\top} E\left(\beta_{t} S_{t}-\beta_{t-1} S_{t-1} \mid \mathcal{F}_{t-1}\right)
$$

for $t=T, \ldots, 1$.

Moreover the optimal value of $V_{0}$ is $C_{0}$, and

$$
E\left(G^{2}\right)=\sum_{t=1}^{T} E\left(\beta_{t}^{2} G_{t}^{2}\right),
$$

where $G_{t}=\varphi_{t}^{\top}\left\{S_{t}-E\left(S_{t} \mid \mathcal{F}_{t-1}\right)\right\}-\left\{C_{t}-E\left(C_{t} \mid \mathcal{F}_{t-1}\right)\right\}, 1 \leq t \leq T$.

Having found the optimal hedging strategy, according to the mean square error criterion, one might ask what the link is between the price given by $C_{0}$, as in Theorem 1 , and the price suggested by the martingale measure method. The answer is given by the following result proven in Appendix D.2.

Corollary 1 For any $t=1 \ldots, T$, set

$$
U_{t}=1-\Delta_{t}^{\top}\left(\Sigma_{t}\right)^{-1} E\left(S_{t}-\beta_{t-1} S_{t-1} / \beta_{t} \mid \mathcal{F}_{t-1}\right) .
$$

Further set $M_{0}=1$ and $M_{t}=U_{t} M_{t-1}, 1 \leq k \leq n$. Then $\left(M_{t}, \mathcal{F}_{t}\right)_{t=0}^{T}$ is a (not necessarily positive) martingale and

$$
\beta_{t-1} C_{t-1}=E\left(\beta_{t} C_{t} U_{t} \mid \mathcal{F}_{t-1}\right)
$$

In particular $\beta C_{t} M_{t}$ is a martingale and $C_{0}=E\left(\beta_{T} C_{T} M_{T} \mid \mathcal{F}_{0}\right)$. Moreover $E\left(\beta_{t} S_{t} U_{t} \mid \mathcal{F}_{t-1}\right)=$ $\beta_{t-1} S_{t-1}$, so $\beta_{t} S_{t} M_{t}$ is a martingale. ${ }^{1}$

\footnotetext{
${ }^{1}$ When the market is complete, there is a unique martingale measure $Q$ and every claim is attainable, so the risk associated with the optimal strategy is zero. Therefore $M_{t}$, as defined in Corollary 1 is positive, and as a by-product of our method, we have an explicit representation of the density of $Q$ with respect to $P$.
} 


\subsubsection{The Markovian case}

If the price process $S$ is Markovian, i.e., the law of $S_{t}$ given $\mathcal{F}_{t-1}$ is $\nu_{t}\left(S_{t-1}, d x\right)$, and if the terminal payoff $C_{T}=C$ only depends on the terminal prices, that is $C=f_{T}\left(S_{T}\right)$, then the Markov property, together with Theorem 1, yield that $C_{t}=f_{t}\left(S_{t}\right)$ and $\varphi_{t}=\psi_{t}\left(S_{t-1}\right)$, where

$$
\begin{aligned}
L_{1 t}(s) & =E\left(S_{t} \mid S_{t-1}=s\right)=\int x \nu_{t}(s, d x), \\
L_{2 t}(s) & =E\left(S_{t} S_{t}^{\top} \mid S_{t-1}=s\right)=\int x x^{\top} \nu_{t}(s, d x), \\
A_{t}(s) & =L_{2 t}(s)-L_{1 t}(s) L_{1 t}(s)^{\top}, \\
\psi_{t}(s) & =A_{t}(s)^{-1} E\left[\left\{S_{t}-L_{1 t}(s)\right\} f_{t}\left(S_{t}\right) \mid S_{t-1}=s\right] \\
& =A_{t}(s)^{-1} \int\left(x-L_{1 t}(s)\right) f_{t}(x) \nu_{t}(s, d x), \\
U_{t}(s, x) & =1-\left(L_{1 t}(s)-\beta_{t-1} s / \beta_{t}\right)^{\top} A_{t}(s)^{-1}\left(x-L_{1 t}(s)\right), \\
f_{t-1}(s) & =\frac{\beta_{t}}{\beta_{t-1}} E\left\{U_{t}\left(s, S_{t}\right) f_{t}\left(S_{t}\right) \mid S_{t-1}=s\right\} \\
& =\frac{\beta_{t}}{\beta_{t-1}} \int U_{t}(s, x) f_{t}(x) \nu_{t}(s, d x) .
\end{aligned}
$$

Note that $E\left(S_{t} \mid \mathcal{F}_{t-1}\right)=L_{1 t}\left(S_{t-1}\right)$ and $\Sigma_{t}=A_{t}\left(S_{t-1}\right)$. Explicit calculations can be done when the returns are assumed to be a finite Markov chain. In most models, one can write $S_{t}=\omega_{t}\left(S_{t-1}, \xi_{t}\right)$ where $\xi_{t}$ is independent of $\mathcal{F}_{t-1}$ and has law $P_{t}$. When $\mu_{t}$ has an infinite support, there are ways to approximate $\psi_{t}$ and $f_{t}$.

The importance of Theorem 1 to the replication problem of hedge funds is obvious, particularly under the Markovian setting. All that is needed is a way to calculate or approximate the value of $f_{0}$ and of the deterministic functions $\psi_{t}(s), f_{t}(s), t=1, \ldots$ In particular $V_{0}=f_{0}$ and $\varphi_{t}=\psi_{t}(s)$ gives the optimal hedging strategy when $S_{t-1}=s$.

\subsubsection{The dynamic trading strategy}

In the Markovian case, one can use the methodology developed by Del Moral et al. (2006) to calculate both the $\varphi_{t}$ 's and the $C_{t}$ 's. The algorithm for implementing the dynamic trading strategy, based on Monte Carlo simulations and linear interpolation, is described in more details in Appendix B. 


\subsection{A comparison between optimal hedging and hedging under Black-Scholes setting}

To compare the two methods, simply take $T=1$ and $r=0$ and $d=1$. In this case, the solution for optimal hedging yields $\varphi^{\star}=\operatorname{Cov}\left\{\Delta S_{1}, C\left(S_{1}\right)\right\} / \operatorname{Var}\left(\Delta S_{1}\right)$, where $\Delta S_{1}=S_{1}-S_{0}$, and $V_{0}^{\star}=E\left\{C\left(S_{1}\right)\right\}-\varphi^{\star} E\left(\Delta S_{1}\right)$.

For the Black-Scholes setting, we have

$$
V_{0}^{B S}=E\left\{C\left(S_{0} e^{\sigma Z-\sigma^{2} / 2}\right)\right\} \quad \text { and } \quad \varphi^{B S}=E\left\{e^{\sigma Z-\sigma^{2} / 2} C^{\prime}\left(S_{0} e^{\sigma Z-\sigma^{2} / 2}\right)\right\},
$$

with $\sigma^{2}=\operatorname{Var}\left\{\log \left(S_{1} / S_{0}\right)\right\}$, where $Z \sim N(0,1)$, provided $C$ is differentiable. See, e.g., Broadie and Glasserman (1996). In general, $\varphi^{\star} \neq \varphi^{B S}$ and $V_{0}^{\star} \neq V_{0}^{B S}$, so

$$
E\left[\left\{V_{1}\left(V_{0}^{\star}, \varphi^{\star}\right)-C\left(S_{1}\right)\right\}^{2}\right]<E\left[\left\{V_{1}\left(V_{0}^{B S}, \varphi^{B S}\right)-C\left(S_{1}\right)\right\}^{2}\right] .
$$

For an analysis of the (discrete) hedging error in a Black-Scholes setting, see, e.g., Wilmott (2006). To illustrate the difference in an hedge funds context, we performed a numerical experiment in which we tried (10 000 times) to reproduce a synthetic fund with centered Gaussian distribution with annual volatility $12 \%$ and correlation $30 \%$ with the portfolio. The distribution of the daily returns of the (portfolio, reserve) pair are modeled by a a mixture of 4 regimes for the daily returns distribution with parameters given in Table 1. With this choice of parameters, it turns out that the associated monthly returns are best modeled by a bivariate Gaussian with parameters are given in Table 2 .

Table 1: Parameters for the Gaussian mixture with 4 regimes used for modeling daily returns

\begin{tabular}{c|r|r|c|c|c}
$\pi_{k}$ & \multicolumn{1}{|c|}{$\mu_{k 1}$} & \multicolumn{1}{c|}{$\mu_{k 2}$} & $\sigma_{1 k}$ & $\sigma_{2 k}$ & $\rho_{k}$ \\
\hline & & & & & \\
0.0956 & 0.0016 & 0.0008 & 0.0039 & 0.0016 & 0.9754 \\
0.4673 & 0.0000 & 0.0002 & 0.0069 & 0.0032 & 0.7981 \\
0.0763 & -0.0003 & -0.0005 & 0.0115 & 0.0054 & 0.6964 \\
0.3607 & 0.0006 & 0.0005 & 0.0037 & 0.0027 & 0.4613 \\
\hline
\end{tabular}

As said previously, we simulated 10000 values of $g\left(R_{0, T}^{(1)}, R_{0, T}^{(2)}\right), \log \left(V_{T}^{\star} / 100\right)$ (under optimal hedging) and $\log \left(V_{T}^{B S} / 100\right)$ (under delta hedging). Some sample characteristics of these three variables are given in Table 3, together with the corresponding true values, while for each dynamic trading method, the estimated mean hedging error and square root mean square error are given in Table 4. 
Table 2: Estimation of the parameters of the Gaussian model compatible with the daily returns

\begin{tabular}{c|c|c|c|c}
$\mu_{1}$ & $\mu_{2}$ & $\sigma_{1}$ & $\sigma_{2}$ & $\rho$ \\
\hline 0.007892797 & 0.0068086 & 0.029334999 & 0.014646356 & 0.700295314 \\
\hline
\end{tabular}

By construction, optimal hedging always produces an hedging error with zero mean. However, this is not the case in general for delta hedging. Note how far the delta hedging method is off the goal of a zero mean of the replicating portfolio, while the optimal hedging error is much smaller.

As our proposed method is optimal for minimizing the square hedging error, it is not surprising that it dominates delta hedging. However, since the theoretical setting is very close to the Black-Scholes setting, all monthly returns being Gaussian, it is worth noting that the square root Mean Square Error of the optimal hedging is $150 \%$ less than the one of the delta hedging.

Finally, the distribution of the respective hedging errors is illustrated in Figure 1. From that graph, it appears that the values of the replication portfolio with the methodology proposed in Kat and Palaro (2005) are almost always smaller than the target values.

Table 3: Replication results based on 10000 trajectories for $g\left(R_{0, T}^{(1)}, R_{0, T}^{(2)}\right)=\log \left(C_{T} / 100\right)$ and $\log \left(V_{T} / 100\right)$ under optimal hedging and delta hedging.

\begin{tabular}{l|c|c|c|c} 
Parameter & True value & $g$ & Optimal hedging & Delta hedging \\
\hline & \multicolumn{3}{|c}{} \\
Mean & 0 & $3.957 \mathrm{E}-07$ & $3.574 \mathrm{E}-07$ & -0.000422735 \\
Std. dev. & 0.034641016 & 0.034957842 & 0.034961135 & 0.034985553 \\
Skewness & 0 & -0.058910418 & -0.064053039 & -0.063978046 \\
Kurtosis & 0 & 0.029916203 & 0.032479236 & 0.032374552 \\
$\rho$ & 0.3 & 0.30283895 & 0.30279462 & 0.30288552
\end{tabular}

\section{Replication of hedge funds}

In this section we will provide some empirical evidence regarding the ability of the model to replicate hedge fund returns. For the sake of parsimony, we will present results for 
Table 4: Replication results based on 10000 trajectories for the payoff $\tilde{g}$ and $\log \left(V_{T} / 100\right)$ under optimal hedging and delta hedging.

\begin{tabular}{l|c|c|c} 
Parameter & Optimal hedging & Delta hedging & $|O H / B S|$ \\
\hline & & \multicolumn{3}{|c}{$\mid$} & \\
Mean hedging error & 0.000004009 & -0.042061101 & 10491.66889 \\
Square root MSE & 0.017861376 & 0.045665732 & 2.556674977
\end{tabular}

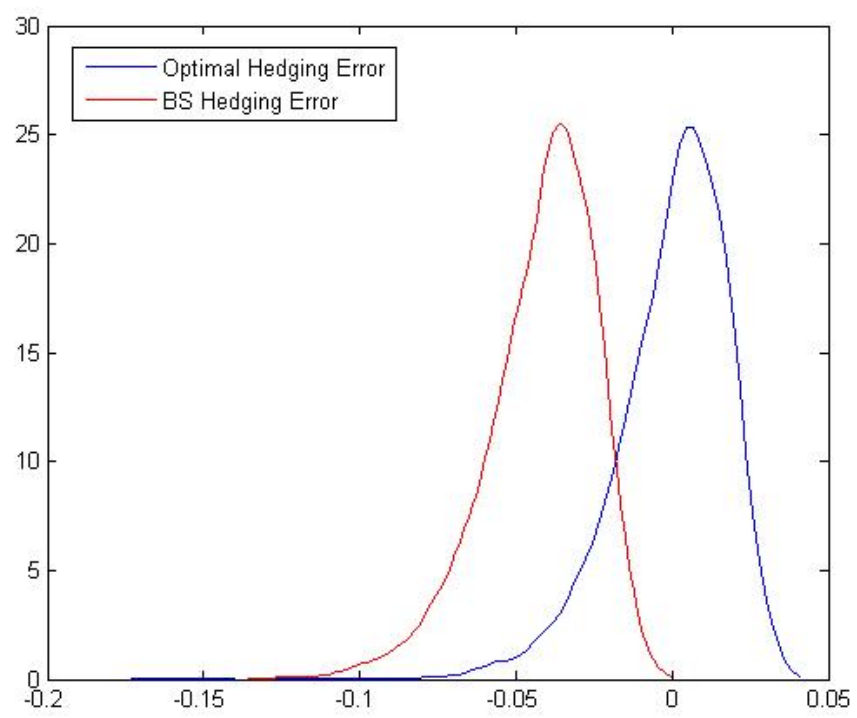

Figure 1: Kernel density estimation of hedging errors for optimal hedging and delta hedging.

the (in-sample) replication of the EDHEC indices and HFR indices. We will look at the models ability to replicate the statistical properties of the monthly returns of the different indices over the ten year period from 01/30/1997 to 12/29/2006 (120 months), as well as for 2 subperiods ranging respectively from 01/30/1997 to 12/29/2001 (59 months) and from $12 / 30 / 2001$ - 12/29/2006 (61 months).

\subsection{Portfolio and Reserve assets}

The first step is top select the assets that will make up the investor portfolio, $S^{(1)}$, and the reserve asset, $S^{(2)}$. Because these two portfolios are dynamically traded on a daily basis, we 
seek very liquid instruments with low transaction costs. We therefore restrict the components of these two assets to be either Futures contracts or Exchange Traded Funds (ETF).

All futures data comes from CRB Trader database. The cash rate is the BBA Libor 1 month rate. Log-returns on futures are calculated from the reinvestment of a rolling strategy in the front contract. The front contract is the nearest to maturity, on the March/June/September/December schedule and is rolled on the first business day of the maturity month at previous close prices. Each future contract is fully collateralized, so that, the total return is the sum the rolling strategy's return and the cash rate. The ETF data is obtained from Bloomberg.

The investor portfolio, which is meant to be a proxy for a typical institutional portfolio, will be an equal-weighted portfolio of S\&P500 futures contracts and 30 year US Treasury Bond futures contracts. In order to illustrate the sensitivity of the methodology to the choice of reserve asset, we will perform the study using two very different reserve assets. The first asset (Reserve 1) is made up of 50\% PowerShares Dynamic Small Cap Value Portfolio, 25\% iShares Lehman 20 Year Treasury Bond Fund and 25\% Citigroup Treasury 10 Year Bond Fund. The second asset (Reserve 2) is an equally weighted portfolio Two Year Treasury Notes, Ten Year Treasury Notes, S\&P500, and Goldman Sachs Commodity Index future contracts.

Table 5 presents some of the statistical properties of our investor portfolio and the two reserve assets for the entire ten year period and the two sub-periods. We report the mean, standard deviation, skewness, robust skewness ${ }^{2}$, kurtosis, robust kurtosis ${ }^{3}$

As explained in Section 3.1, we have chosen to model the daily returns of the pairs (portfolio, reserve) by bivariate Gaussian mixtures with $m$ regimes, denoted by BGM(m).

In Table 6, the distributions of the daily and monthly returns for the (portfolio, reserve) pairs are given, over the three time periods. These results were obtained by using the estimation and goodness-of-it procedures described in Section 3.1.3.

It may seems odd at first that the model for the joint monthly returns is a (bivariate) Gaussian mixture with fewer regimes than for the daily returns. However, as explained in Remark 3.1, it is quite normal. In fact, in view of the central limit theorem, the number of regimes would possibly be 1 if we were to consider returns over a two months period.

\subsection{Hedge fund indices}

For the sake of comparison, we chose to replicate the 13 EDHEC indices and the 22 HFRI indices. According to the procedures described in Sections 3.2 and 3.3, the marginal distri-

\footnotetext{
${ }^{2}$ Defined by $\{E(X)-Q(1 / 2)\} / E\{|X-Q(1 / 2)|\}$, where $Q_{\alpha}$ is the $\alpha$-quantile.

${ }^{3}$ Defined by $0.09+\{Q(.975)-Q(.025)\} /\{Q(.75)-Q(.25)\}$.
} 
Table 5: Summary statistics for the portfolio and the reserve assets over the three time periods.

\begin{tabular}{|c|c|c|c|c|}
\hline Asset & Statistics & Period $1(97-06)$ & Period $2(97-01)$ & Period $3(02-06)$ \\
\hline \multirow{6}{*}{ Portfolio } & Mean & 0.0035 & 0.0047 & 0.0024 \\
\hline & S.Dev & 0.0244 & 0.0289 & 0.0192 \\
\hline & Skew & -0.2150 & -0.2697 & -0.2482 \\
\hline & R. Sk & -0.0813 & -0.2665 & -0.1097 \\
\hline & Kurt & 3.2109 & 2.6942 & 3.6637 \\
\hline & R. Kurt & 3.2467 & 2.7483 & 3.6386 \\
\hline \multirow{7}{*}{ Reserve 1} & Mean & 0.0094 & 0.0095 & 0.0093 \\
\hline & S.Dev & 0.0225 & 0.0260 & 0.0187 \\
\hline & Skew & 0.3006 & 0.5346 & -0.3480 \\
\hline & R. Sk & 0.0362 & 0.0552 & 0.0159 \\
\hline & Kurt & 5.0025 & 5.0399 & 3.2161 \\
\hline & R. Kurt & 3.2419 & 4.0561 & 2.9244 \\
\hline & Corr. with Port. & 0.6749 & 0.7054 & 0.6206 \\
\hline \multirow{7}{*}{ Reserve 2} & Mean & (0.0031 & (20.0016 & 0.0047 \\
\hline & S.Dev & 0.0195 & 0.0219 & 0.0168 \\
\hline & Skew & 0.0338 & 0.3193 & -0.3886 \\
\hline & R. Sk & -0.0891 & -0.0161 & -0.2345 \\
\hline & Kurt & 3.4509 & 3.3083 & 3.7213 \\
\hline & R. Kurt & 3.3207 & 3.3894 & 3.4959 \\
\hline & Corr. with Port. & 0.6040 & 0.7231 & 0.3989 \\
\hline
\end{tabular}

Table 6: Distribution of the daily and monthly returns for the two pairs (portfolio, reserve), over the three time periods.

\begin{tabular}{|c|c|c|c|c|c|c|}
\hline \multirow[t]{2}{*}{ Returns } & \multicolumn{2}{|c|}{ Period 1 (97-06) } & \multicolumn{2}{|c|}{ Period $2(97-01)$} & \multicolumn{2}{|c|}{ Period $3(02-06)$} \\
\hline & Reserve 1 & Reserve 2 & Reserve 1 & Reserve 2 & Reserve 1 & Reserve 2 \\
\hline Daily & $\overline{\mathrm{BGM}(5)}$ & BGM(5) & $\overline{\mathrm{BGM}(5)}$ & $\overline{\mathrm{BGM}(5)}$ & $\overline{\mathrm{BGM}(3)}$ & $\overline{\overline{B G M}(4)}$ \\
\hline Monthly & $\operatorname{BGM}(2)$ & $\operatorname{BGM}(2)$ & $\operatorname{BGM}(4)$ & $\operatorname{BGM}(2)$ & $\operatorname{BGM}(2)$ & $\operatorname{BGM}(3)$ \\
\hline
\end{tabular}

bution $F_{3}$ and the copula $C_{1,3}$ were estimated for each hedge fund index.

For the marginal distributions, we considered (univariate) Gaussian mixtures with $m$ regimes, denoted $\mathrm{GM}(\mathrm{m})$ and Johnson distribution. For the copula families, we selected the Gaussian, Student, Clayton, Frank and Gumbel. In each case, we estimated Kendall's tau, 
which measures the dependence between the hedge fund returns and the portfolio returns. Except for the Student copula, which is dependent on two parameters, the other families only depend on one parameter.

The best fitting models are displayed in Tables 8-10.

\subsection{Performance of the replication}

There are two important issues that need to be addressed when analyzing the models ability to replicate hedge fund returns. The first issue concerns the models ability to effectively replicate hedge fund indices. The second issue pertains to the choice of the reserve asset and it's impact on the models performance.

To study the effectiveness of the replication strategies, there are two main factors to consider: the initial investment $V_{0}$ that is required to replicate each index as well as the actual quality of the replication. In order to obtain the payoff distribution of the hedge fund indices, we follow the approach used by Kat and Palaro (2005)- we calculate the monthly returns assuming an investment of 100 at the beginning of each month. Therefore, if the value $V_{0}$ of the replicating strategy is below 100, this would lead us to conclude that the replicating strategy offers a cheaper alternative to the hedge fund index, and therefore is the better investment choice. This analysis can however be misleading if we do not also examine the precision of the replication strategy. Before dismissing the hedge fund indices as poor-performers, we need to properly evaluate whether the properties of the replication strategies and hedge fund indices are truly the same. A proper examination of both the cost and the precision of the replication strategy is fundamental before any strong conclusion can be drawn about the model's ability to replicate hedge fund indices.

Then arises the question of the reserve asset. Does the reserve asset impact the performance of the model, and if so does it affect only $V_{0}$ or also the ability of the model to replicate the statistical properties of the hedge fund indices? In other words, does the choice of reserve asset impact the performance measure and/or for the quality of the replication?

Tables 11-13 present the values of $V_{0}$ for the HFRI and EDHEC hedge fund indices. It is quite clear that even without correcting for the well documented biases in hedge fund indices, the replicating strategies still out-perform a large number of the hedge fund indices over the entire period as well as over the two sub-periods. In order to show that the replication strategies are effectively reproducing the statistical properties of the hedge fund indices, Figures 2-6 present the target mean, volatility, Kendall's tau, skewness and kurtosis of the indices as well as those for the replication strategies. It is quite clear that independently of the period that is considered, the volatility and Kendall's tau are reproduced with great precision. It is important to note that the only moment that is sensitive to the choice of reserve asset is the return of the replication strategy - the other moments as well as the 
dependence coefficient appear to be insensitive to the choice of reserve asset. Our results clearly indicate that the reserve asset plays a role in the measure of performance, $V_{0}$, but it has almost no effect on the quality of the replication.

Table 7: Regression of EDHEC and HFRI indices returns with the replication returns (for reserve assets 1-2) for the following target parameters: volatility, skewness, robust skewness, kurtosis, robust kurtosis, Kendall's tau and Pearson's rho.

\begin{tabular}{ccccccc}
$\begin{array}{c}\text { Period: }(1997-2006) \\
\text { Target }\end{array}$ & Intercept & Slope & $R^{2}(\%)$ & Intercept & Slope & $R^{2}(\%)$ \\
\hline \hline Volatility & 0.000624738 & 0.997485421 & 99.38 & 0.000132117 & 1.034882095 & 99.38 \\
Skewness & -1.21833672 & 1.135438624 & 63.48 & -0.660897414 & 1.017065756 & 78.82 \\
Robust Skew & 0.005285212 & 0.591422785 & 38.74 & 0.049971694 & 0.845539485 & 68.79 \\
Kurtosis & 1.427089662 & 1.320048662 & 26.05 & 1.738641971 & 1.116543294 & 79.34 \\
Robust Kurt & 2.057766094 & 0.48321167 & 36.19 & 1.800169291 & 0.491547026 & 34.31 \\
Kendall's Tau & 0.040820382 & 1.009779392 & 98.80 & 0.034979443 & 1.024409652 & 99.36 \\
Pearson's Rho & 0.031939885 & 1.046644073 & 95.80 & 0.030103056 & 1.064383569 & 96.32 \\
\hline
\end{tabular}

\begin{tabular}{ccccccc} 
Period: (1997-2001) & \multicolumn{3}{c}{ Reserve 1} \\
Target & Intercept & Slope & $R^{2}(\%)$ & Intercept & Slope & $R^{2}(\%)$ \\
\hline \hline Volatility & 0.000246245 & 0.999597258 & 98.15 & 0.000303651 & 1.026011825 & 98.27 \\
Skewness & -0.58025733 & 0.917232282 & 32.10 & -0.86585092 & 1.542889847 & 65.17 \\
Robust Skew & 0.044192227 & 0.916761936 & 56.14 & -0.00965482 & 0.729453739 & 54.08 \\
Kurtosis & 5.581125649 & 0.675401646 & 15.27 & 2.081736175 & 1.733323643 & 20.62 \\
Robust Kurt & -0.85346256 & 1.451214328 & 67.35 & -0.58104505 & 1.267471264 & 63.20 \\
Kendall's Tau & 0.0254162 & 1.019450292 & 98.52 & 0.020171502 & 1.016297547 & 99.18 \\
Pearson's Rho & 0.056163582 & 1.022429795 & 91.53 & 0.021180004 & 1.06516375 & 94.76 \\
\hline
\end{tabular}

\begin{tabular}{ccccccc} 
Period: $(2002-2006)$ & \multicolumn{3}{c}{ Reserve 2} \\
Target & Intercept & Slope & $R^{2}(\%)$ & Intercept & Slope & $R^{2}(\%)$ \\
\hline \hline Volatility & -0.00015482 & 0.987878677 & 99.84 & 0.000145601 & 0.984626992 & 99.59 \\
Skewness & -0.07264573 & 1.035201227 & 83.03 & 0.045240922 & 1.104989802 & 80.44 \\
Robust Skew & 0.004977508 & 0.816341527 & 53.79 & 0.068954198 & 1.033664472 & 61.45 \\
Kurtosis & 1.26397635 & 0.768109088 & 35.44 & 0.536774019 & 0.93664374 & 67.14 \\
Robust Kurt & 1.471815049 & 0.540835024 & 45.90 & 1.421018259 & 0.566191474 & 26.63 \\
Kendall's Tau & -0.00043248 & 1.069855948 & 98.96 & 0.019777659 & 1.034958883 & 98.97 \\
Pearson's Rho & 0.059853575 & 1.027479829 & 91.93 & 0.119157269 & 1.054127939 & 91.77 \\
\hline
\end{tabular}

In order to further examine the model's ability to replicate the statistical properties 
of the hedge fund indices, Table 7 presents the results obtained by regressing the statistical properties of the replication portfolios against the estimated parameters of both EDHEC and HFRI indices for the three samples periods. If the replications were perfect, the slope would then be 1 and the intercept would be 0 . As one can see, the fit is very impressive for both reserve assets. The volatility and dependence measures (Kendal's tau and Spearman's Rho) are perfectly replicated, and the regression coefficients for the higher moments, although not perfect, support the model's ability to replicate the statistical properties of hedge fund returns.

The final stage of the analysis consists of breaking down the costs and other potential sources of error associated with the dynamic replicating strategy. We quantify three potential costs/errors associated with our methodology. The first is the transaction costs related to the dynamic trading; the second is the rounding error that results from not being able to trade fractions of futures contracts; the third, and most significant, is the profit/loss that is due to the hedging error of the discrete hedging strategy.

The transaction costs are assumed to be 1 basis point for the sale/purchase of all futures contracts. Obviously, the amount of trading required to replicate the different indices can vary substantially. In table 14 we present the average monthly transaction costs (in terms of basis points) incurred for each replicating portfolio over the whole sample period. Note that the average monthly transaction costs for the replication strategies is approximately 5 basis points.

The rounding error that results from the inability to buy or sell fractions of futures contracts depends very much on the size of the replication portfolio and this error tends to zero as the portfolio increases in size. For a replicating strategy with $\$ 100$ Million invested, the average monthly rounding error is approximately 1 basis point.

Finally, we calculate replicating errors, that is the average difference between the value of the replicating strategy and the value of the hedge fund index. The results are presented in Tables 15-17. Note that the average monthly hedging error on all replications as defined in Equation 5 is around 3 basis points.

\section{Conclusion}

In this paper, we implement a multi-variate extension of Dybvig (1988) Payoff Distribution Model that can be used to replicate not only the marginal distribution of hedge fund returns but also their dependence with other asset classes. In addition to proposing ways to overcome the hedging and compatibility inconsistencies in Kat and Palaro (2005) we extend the results of Schweizer (1995) and adapt American options pricing techniques to evaluate the model and also derive an optimal dynamic trading (hedging) strategy. In section 5.2 we demonstrate the superiority of the hedging algorithm that is used to generate the dynamic replicating strategy. 
We successfully replicate the statistical properties of the HFRI and EDHEC indices over the period from 1997-2006, as well as for two 60 month sub-periods. Even without correcting for the well-documented biases in hedge fund index returns, the indices can be readily replicated using this methodology. The volatility and the dependence coefficients are replicated with great precision; the skewness and kurtosis are also captured by the model, however with slightly less accuracy.

Contrary to the conclusions put forth by recent studies at EDHEC (?) and Northwater (?), the choice of reserve asset does not impact the model's ability to replicate the statistical properties of the indices. The choice of reserve asset only impacts the initial cost of investing in the replicating portfolio (and hence only impacts the return of the replicating strategy). This is not to say that the return generated by the model is not important, however it is not a measure of the model's success. One must dissociate the technical issues of the replicating methodology (i.e how to best model the returns and solve for the optimal trading strategy) from the choice of the reserve asset. Our contribution is to provide a robust framework for the replication methodology, and address the technical shortcomings of the much publicized research of Kat and Palaro.

As is the case with any investment strategy, the returns depend on the choice of assets. The results in this paper indicate, however, that it is not necessary to select the best performing assets over the sample period in order to replicate and outperform the hedge fund indices. In fact, we show that by using run-of-the-mill exposures in our reserve asset we can nonetheless outperform the majority of hedge fund indices. We purposely selected two reserve assets that have exposures to different yet common market premia over the sample period, and we find that both reserve assets outperform a large percentage of the indices. (reserve 1 being the better of the two). We also find that the EDHEC indices, which are subject to less significant biases, are more easy to replicate that the HFRI indices. It is important to remember that we are comparing an investable trading strategy to non-investable indices- the actual return we would anticipate from investing in a hedge fund index would be considerably lower than the "non-investable" index returns used in this study. Our results reinforce the notion that on aggregate, hedge funds are on aggregate simply repackaging beta returns.

\section{References}

Agarwal, V. and Naik, N. (2004). Risks and portfolio decisions involving hedge funds. Review of Financial Studies, 17:63-98.

Amin, G. and Kat, H. (2003). Hedge fund performanc 1990-2000: Do the "money machines" rellay add value. Journal of Financial and Quantitative Analysis, 38(2):251-275. 
Broadie, M. and Glasserman, P. (1996). Estimating security price derivatives using simulation. Management Science, 42:260-285.

Chen, Y. and Liang, B. (2006). Do market timing hedge funds time the market? Technical report, Carroll School of Management, Boston College.

Del Moral, P., Rémillard, B., and Rubenthaler, S. (2006). Monte Carlo approximations of American options. Technical report, GERAD.

Dempster, A. P., Laird, N. M., and Rubin, D. B. (1977). Maximum likelihood from incomplete data via the EM algorithm. J. Roy. Statist. Soc. Ser. B, 39:1-38.

Durbin, J. (1973). Weak convergence of the sample distribution function when parameters are estimated. Ann. Statist., 1(2):279-290.

Dybvig, P. (1988). Distributional analysis of portfolio choice. The Journal of Business, 61(3):369-393.

Fung, W. and Hsieh, D. (2001). The risk in hedge fund stategies: Theory and evidence from trend followers. Review of Financial Studies, 14:313-341.

Genest, C., Ghoudi, K., and Rivest, L.-P. (1995). A semiparametric estimation procedure of dependence parameters in multivariate families of distributions. Biometrika, 82:543-552.

Genest, C., Quessy, J.-F., and Rémillard, B. (2006). Goodness-of-fit procedures for copula models based on the integral probability transformation. Scand. J. Statist., 33:337-366.

Genest, C. and Rémillard, B. (2005). Validity of the parametric bootsrap for goodness-of-fit testing in semiparametric models. Technical Report G-2005-51, GERAD.

Genest, C., Rémillard, B., and Beaudoin, D. (2007). Omnibus goodness-of-fit tests for copulas: A review and a power study. Insurance Math. Econom., 40:in press.

Hasanhodzic, J. and Lo, A. W. (2007). Can hedge-fund returns be replicated?: The linear case. Journal of Investment Management, 5(2):5-45.

Kat, H. and Palaro, H. (2005). Who needs hedge funds? A copula-based approach to hedge fund return replication. Technical report, Cass Business School, City University.

Kazemi, H. and Schneeweis, T. (2003). Conditional performance of hedge funds. Technical report, CISDM.

Mitchell, M. and Pulvino, T. (2001). Characteristics of risk and return in risk arbitrage. Journal of Finance, LVI:2135-2175. 
Nelsen, R. B. (1999). An introduction to copulas, volume 139 of Lecture Notes in Statistics. Springer-Verlag, New York.

Rosenblatt, M. (1952). Remarks on a multivariate transformation. Ann. Math. Stat., 23:470472.

Schweizer, M. (1995). Variance-optimal hedging in discrete time. Math. Oper. Res., 20(1):132.

Stute, W., Gonzáles Manteiga, W., and Presedo Quindimil, M. (1993). Bootstrap based goodness-of-fit tests. Metrika, 40:243-256.

Wilmott, P. (2006). Paul Wilmott on Quantitative Finance, volume 3. John Wiley \& Sons. 
Table 8: Marginal distribution, copula and Kendall's tau for entire period (1997-2006).

\begin{tabular}{|c|c|c|c|}
\hline Fund & Marginal & Copula & Kendall's tau \\
\hline EDHEC-Convertible Arbitrage & $\mathrm{GM}(3)$ & Frank & 0.0927 \\
\hline EDHEC-CTA Global & $\operatorname{GM}(2)$ & Gumbel & 0.0552 \\
\hline EDHEC-Distressed Securities & $\operatorname{GM}(2)$ & Clayton & 0.2311 \\
\hline EDHEC-Emerging Markets & Johnson & Frank & 0.3394 \\
\hline EDHEC-Equity Market Neutral & GM(2) & Frank & 0.2302 \\
\hline EDHEC-Event Driven & GM(3) & Frank & 0.3724 \\
\hline EDHEC-Fixed Income Arbitrage & GM(3) & Frank & 0.0997 \\
\hline EDHEC-Global Macro & GM(3) & Frank & 0.3316 \\
\hline EDHEC-Long/Short Equity & GM(2) & Student & 0.4529 \\
\hline EDHEC-Merger Arbitrage & GM(2) & Frank & 0.2956 \\
\hline EDHEC-Relative Value & $\operatorname{GM}(3)$ & Gaussian & 0.3324 \\
\hline EDHEC-Short Selling & $\operatorname{GM}(2)$ & Frank & -0.4636 \\
\hline EDHEC-Funds of Funds & GM(4) & Gaussian & 0.3536 \\
\hline HFRI Convertible Arbitrage Index & GM(3) & Frank & 0.1048 \\
\hline HFRI Distressed Securities Index & GM(3) & Clayton & 0.2160 \\
\hline HFRI Emerging Markets (Total) & Johnson & Student & 0.3269 \\
\hline HFRI Equity Hedge Index & GM(2) & Clayton & 0.4530 \\
\hline HFRI Equity Market Neutral Index & GM(3) & Frank & 0.1345 \\
\hline HFRI Equity Non-Hedge Index & GM(3) & Student & 0.4770 \\
\hline HFRI Event-Driven Index & GM(3) & Clayton & 0.3700 \\
\hline HFRI Fixed Income (Total) & $\mathrm{GM}(3)$ & Frank & 0.3168 \\
\hline HFRI Fixed Income: Arbitrage Index & $\operatorname{GM}(3)$ & Ind. & 0 \\
\hline HFRI Fixed Income: High Yield Index & $\operatorname{GM}(2)$ & Student & 0.2036 \\
\hline HFRI FOF: Conservative Index & Johnson & Frank & 0.3021 \\
\hline HFRI FOF: Diversified Index & GM(3) & Frank & 0.2945 \\
\hline HFRI FOF: Market Defensive Index & $\operatorname{GM}(2)$ & Frank & 0.1020 \\
\hline HFRI FOF: Strategic Index & GM(3) & Frank & 0.3555 \\
\hline HFRI FOF Composite Index & GM(3) & Frank & 0.3327 \\
\hline HFRI FOF Composite Index (Off.) & GM(3) & Frank & 0.3180 \\
\hline HFRI Fund Weighted Composite Index & GM(3) & Clayton & 0.4403 \\
\hline HFRI Macro Index & GM(2) & Clayton & 0.2364 \\
\hline HFRI Merger Arbitrage Index & GM(3) & Frank & 0.2568 \\
\hline HFRI Regulation D Index & GM(3) & Gaussian & 0.2210 \\
\hline HFRI Relative Value Arbitrage Index & GM(3) & Gaussian & 0.2567 \\
\hline HFRI Short Selling Index & GM(3) & Frank & -0.4520 \\
\hline
\end{tabular}


Table 9: Marginal distribution, copula and Kendall's tau for first sub-period (1997-2001).

\begin{tabular}{|c|c|c|c|}
\hline Fund & Marginal & Copula & Kendall's tau \\
\hline EDHEC-Convertible Arbitrage & $\overline{\mathrm{GM}(3)}$ & Gumbel & 0.0777 \\
\hline EDHEC-CTA Global & $\operatorname{GM}(2)$ & Ind. & 0 \\
\hline EDHEC-Distressed Securities & $\operatorname{GM}(3)$ & Clayton & 0.2309 \\
\hline EDHEC-Emerging Markets & $\operatorname{GM}(3)$ & Frank & 0.3241 \\
\hline EDHEC-Equity Market Neutral & $\operatorname{GM}(2)$ & Gaussian & 0.3691 \\
\hline EDHEC-Event Driven & Johnson & Clayton & 0.3793 \\
\hline EDHEC-Fixed Income Arbitrage & $\operatorname{GM}(3)$ & Frank & 0.1268 \\
\hline EDHEC-Global Macro & $\operatorname{GM}(3)$ & Frank & 0.4198 \\
\hline EDHEC-Long/Short Equity & $\operatorname{GM}(2)$ & Frank & 0.4868 \\
\hline EDHEC-Merger Arbitrage & GM(4) & Gumbel & 0.2951 \\
\hline EDHEC-Relative Value & GM(3) & Clayton & 0.3454 \\
\hline EDHEC-Short Selling & GM(2) & Frank & -0.4695 \\
\hline EDHEC-Funds of Funds & GM(2) & Frank & 0.3934 \\
\hline HFRI Convertible Arbitrage Index & GM(3) & Frank & 0.1011 \\
\hline HFRI Distressed Securities Index & $\operatorname{GM}(3)$ & Gaussian & 0.1939 \\
\hline HFRI Emerging Markets (Total) & $\operatorname{GM}(3)$ & Frank & 0.3148 \\
\hline HFRI Equity Hedge Index & $\operatorname{GM}(2)$ & Frank & 0.4880 \\
\hline HFRI Equity Market Neutral Index & $\operatorname{GM}(2)$ & Frank & 0.1607 \\
\hline HFRI Equity Non-Hedge Index & Johnson & Frank & 0.4962 \\
\hline HFRI Event-Driven Index & $\operatorname{GM}(3)$ & Frank & 0.3461 \\
\hline HFRI Fixed Income (Total) & $\operatorname{GM}(3)$ & Frank & 0.3078 \\
\hline HFRI Fixed Income: Arbitrage Index & $\operatorname{GM}(3)$ & Ind. & 0 \\
\hline HFRI Fixed Income: High Yield Index & $\operatorname{GM}(3)$ & Frank & 0.2367 \\
\hline HFRI FOF: Conservative Index & GM(3) & Frank & 0.3310 \\
\hline HFRI FOF: Diversified Index & Johnson & Frank & 0.2915 \\
\hline HFRI FOF: Market Defensive Index & GM(3) & Frank & 0.1257 \\
\hline HFRI FOF: Strategic Index & GM(3) & Frank & 0.3600 \\
\hline HFRI FOF Composite Index & GM(2) & Frank & 0.3427 \\
\hline HFRI FOF Composite Index (Off.) & $\operatorname{GM}(2)$ & Frank & 0.3276 \\
\hline HFRI Fund Weighted Composite Index & GM(3) & Frank & 0.4567 \\
\hline HFRI Macro Index & GM(2) & Clayton & 0.2975 \\
\hline HFRI Merger Arbitrage Index & Johnson & Gumbel & 0.2285 \\
\hline HFRI Regulation D Index & GM(3) & Gaussian & 0.2736 \\
\hline HFRI Relative Value Arbitrage Index & GM(3) & Frank & 0.2705 \\
\hline HFRI Short Selling Index & GM(2) & Frank & -0.4402 \\
\hline
\end{tabular}


Table 10: Marginal distribution, copula and Kendall's tau for second sub-period (2002-2006).

\begin{tabular}{|c|c|c|c|}
\hline Fund & Marginal & Copula & Kendall's tau \\
\hline EDHEC-Convertible Arbitrage & $\mathrm{GM}(3)$ & Gaussian & 0.0885 \\
\hline EDHEC-CTA Global & $\operatorname{GM}(2)$ & Frank & 0.0743 \\
\hline EDHEC-Distressed Securities & $\operatorname{GM}(2)$ & Gaussian & 0.2224 \\
\hline EDHEC-Emerging Markets & $\operatorname{GM}(3)$ & Frank & 0.2710 \\
\hline EDHEC-Equity Market Neutral & Johnson & Frank & 0.0896 \\
\hline EDHEC-Event Driven & Johnson & Gaussian & 0.3052 \\
\hline EDHEC-Fixed Income Arbitrage & $\operatorname{GM}(3)$ & Ind. & 0 \\
\hline EDHEC-Global Macro & $\operatorname{GM}(2)$ & Gaussian & 0.1987 \\
\hline EDHEC-Long/Short Equity & $\operatorname{GM}(2)$ & Clayton & 0.3377 \\
\hline EDHEC-Merger Arbitrage & $\operatorname{GM}(3)$ & Clayton & 0.3126 \\
\hline EDHEC-Relative Value & $\operatorname{GM}(2)$ & Clayton & 0.2973 \\
\hline EDHEC-Short Selling & $\operatorname{GM}(2)$ & Frank & -0.4266 \\
\hline EDHEC-Funds of Funds & Johnson & Clayton & 0.2470 \\
\hline HFRI Convertible Arbitrage Index & $\operatorname{GM}(3)$ & Gumbel & 0.0743 \\
\hline HFRI Distressed Securities Index & $\operatorname{GM}(2)$ & Clayton & 0.2109 \\
\hline HFRI Emerging Markets (Total) & $\operatorname{GM}(3)$ & Frank & 0.2797 \\
\hline HFRI Equity Hedge Index & $\operatorname{GM}(3)$ & Frank & 0.2993 \\
\hline HFRI Equity Market Neutral Index & $\operatorname{GM}(2)$ & Frank & 0.0874 \\
\hline HFRI Equity Non-Hedge Index & $\operatorname{GM}(2)$ & Frank & 0.3687 \\
\hline HFRI Event-Driven Index & $\operatorname{GM}(3)$ & Gaussian & 0.3377 \\
\hline HFRI Fixed Income (Total) & $\operatorname{GM}(3)$ & Gaussian & 0.2303 \\
\hline HFRI Fixed Income: Arbitrage Index & $\operatorname{GM}(3)$ & Ind. & 0 \\
\hline HFRI Fixed Income: High Yield Index & $\operatorname{GM}(2)$ & Gumbel & 0.1311 \\
\hline HFRI FOF: Conservative Index & $\operatorname{GM}(2)$ & Frank & 0.2164 \\
\hline HFRI FOF: Diversified Index & $\operatorname{GM}(2)$ & Clayton & 0.2437 \\
\hline HFRI FOF: Market Defensive Index & $\operatorname{GM}(2)$ & Frank & 0.0831 \\
\hline HFRI FOF: Strategic Index & $\operatorname{GM}(3)$ & Clayton & 0.2885 \\
\hline HFRI FOF Composite Index & $\operatorname{GM}(2)$ & Clayton & 0.2383 \\
\hline HFRI FOF Composite Index (Off.) & $\operatorname{GM}(2)$ & Clayton & 0.2164 \\
\hline HFRI Fund Weighted Composite Index & $\operatorname{GM}(2)$ & Frank & 0.3243 \\
\hline HFRI Macro Index & $\operatorname{GM}(2)$ & Gumbel & 0.0787 \\
\hline HFRI Merger Arbitrage Index & $\operatorname{GM}(2)$ & Clayton & 0.2984 \\
\hline HFRI Regulation D Index & Johnson & Clayton & 0.1552 \\
\hline HFRI Relative Value Arbitrage Index & $\operatorname{GM}(2)$ & Clayton & 0.2328 \\
\hline HFRI Short Selling Index & $\operatorname{GM}(2)$ & Frank & -0.4319 \\
\hline
\end{tabular}


Table 11: Initial investment $V_{0}$ in the replication of EDHEC and HFRI indices for both reserve assets over the entire period (1997-2006).

\begin{tabular}{lcc}
\multicolumn{1}{c}{ Fund } & \multicolumn{2}{c}{$V_{0}$} \\
& Reserve 1 & Reserve 2 \\
\hline EDHEC-Convertible Arbitrage & 99.88746927 & 100.3546058 \\
EDHEC-CTA Global & 99.22395238 & 100.2822217 \\
EDHEC-Distressed Securities & 100.0433158 & 100.5343205 \\
EDHEC-Emerging Markets & 99.20994993 & 100.5118262 \\
EDHEC-Equity Market Neutral & 100.0923959 & 100.3305248 \\
EDHEC-Event Driven & 99.99904541 & 100.5027729 \\
EDHEC-Fixed Income Arbitrage & 99.68524183 & 100.0620038 \\
EDHEC-Global Macro & 99.83012861 & 100.4453958 \\
EDHEC-Long/Short Equity & 99.91948345 & 100.5253251 \\
EDHEC-Merger Arbitrage & 99.94738788 & 100.3347095 \\
EDHEC-Relative Value & 100.044295 & 100.3582369 \\
EDHEC-Short Selling & 97.91881695 & 99.96879961 \\
EDHEC-Funds of Funds & 99.88679097 & 100.4167799 \\
\hline Percentage of $V_{0}$ under 100\$ & $76.92 \%$ & $7.69 \%$ \\
\hline \hline HFRI Convertible Arbitrage Index & 99.9104685 & 100.321649 \\
HFRI Distressed Securities Index & 99.9100765 & 100.446987 \\
HFRI Emerging Markets (Total) & 99.1617091 & 100.497154 \\
HFRI Equity Hedge Index & 99.760536 & 100.537810 \\
HFRI Equity Market Neutral Index & 99.8160615 & 100.178244 \\
HFRI Equity Non-Hedge Index & 99.2694693 & 100.529065 \\
HFRI Event-Driven Index & 99.8678282 & 100.443743 \\
HFRI Fixed Income (Total) & 99.8533463 & 100.180401 \\
HFRI Fixed Income: Arbitrage Index & 99.4744962 & 99.9612590 \\
HFRI Fixed Income: High Yield Index & 99.4606113 & 100.118320 \\
HFRI FOF: Conservative Index & 99.8019766 & 100.171418 \\
HFRI FOF: Diversified Index & 99.5428340 & 100.224120 \\
HFRI FOF: Market Defensive Index & 99.6295097 & 100.290348 \\
HFRI FOF: Strategic Index & 99.3496291 & 100.310468 \\
HFRI FOF Composite Index & 99.6186407 & 100.240115 \\
HFRI FOF Composite Index (Off.) & 99.4353982 & 100.150926 \\
HFRI Fund Weighted Composite Index & 99.7328707 & 100.309632 \\
HFRI Macro Index & 99.6917718 & 100.369990 \\
HFRI Merger Arbitrage Index & 99.8584340 & 100.285088 \\
HFRI Regulation D Index & 99.9386375 & 100.681884 \\
HFRI Relative Value Arbitrage Index & 100.055301 & 100.346992 \\
HFRI Short Selling Index & 97.5229297 & 99.8979799 \\
\hline Percentage of V0 under 100\$ & $95.45 \%$ & $9.09 \%$ \\
\hline & & \\
\hline
\end{tabular}


Table 12: Initial investment $V_{0}$ in the replication of EDHEC and HFRI indices for both reserve assets for first sub-period (1997-2001).

\begin{tabular}{lcc}
\multicolumn{1}{c}{ Fund } & \multicolumn{2}{c}{$V_{0}$} \\
& Reserve 1 & Reserve 2 \\
\hline EDHEC-Convertible Arbitrage & 100.2944853 & 100.8987467 \\
EDHEC-CTA Global & 99.46788172 & 100.9472588 \\
EDHEC-Distressed Securities & 99.90059626 & 100.720508 \\
EDHEC-Emerging Markets & 98.69192451 & 100.9835661 \\
EDHEC-Equity Market Neutral & 100.3954179 & 100.7210641 \\
EDHEC-Event Driven & 100.0609365 & 100.868357 \\
EDHEC-Fixed Income Arbitrage & 99.59077798 & 100.2017969 \\
EDHEC-Global Macro & 99.97142407 & 100.979203 \\
EDHEC-Long/Short Equity & 100.1375749 & 101.127196 \\
EDHEC-Merger Arbitrage & 100.2331299 & 100.7861365 \\
EDHEC-Relative Value & 100.2203665 & 100.6965085 \\
EDHEC-Short Selling & 99.03421453 & 102.1095181 \\
EDHEC-Funds of Funds & 99.96160577 & 100.9516279 \\
\hline Percentage of V $V_{0}$ under 100\$ & $53.84 \%$ & $0.00 \%$ \\
\hline \hline HFRI Convertible Arbitrage Index & 100.2829484 & 100.8055676 \\
HFRI Distressed Securities Index & 99.72936197 & 100.6646377 \\
HFRI Emerging Markets (Total) & 98.09524276 & 100.9525596 \\
HFRI Equity Hedge Index & 100.056951 & 101.5042088 \\
HFRI Equity Market Neutral Index & 100.038409 & 100.6734399 \\
HFRI Equity Non-Hedge Index & 99.05531596 & 101.2392224 \\
HFRI Event-Driven Index & 99.97242706 & 100.980233 \\
HFRI Fixed Income (Total) & 99.75412401 & 100.3504572 \\
HFRI Fixed Income: Arbitrage Index & 99.3254573 & 100.0324407 \\
HFRI Fixed Income: High Yield Index & 99.31890751 & 100.1544936 \\
HFRI FOF: Conservative Index & 99.86644524 & 100.4768867 \\
HFRI FOF: Diversified Index & 99.52279888 & 100.9361689 \\
HFRI FOF: Market Defensive Index & 99.80508973 & 100.7630553 \\
HFRI FOF: Strategic Index & 99.28992499 & 100.9862717 \\
HFRI FOF Composite Index & 99.60846434 & 100.7634087 \\
HFRI FOF Composite Index (Off.) & 99.36188049 & 100.7094413 \\
HFRI Fund Weighted Composite Index & 99.75155852 & 100.9238131 \\
HFRI Macro Index & 99.76812518 & 100.8842713 \\
HFRI Merger Arbitrage Index & 100.1469401 & 100.7111258 \\
HFRI Regulation D Index & 100.5815208 & 101.5412257 \\
HFRI Relative Value Arbitrage Index & 100.1412334 & 100.6153432 \\
HFRI Short Selling Index & 98.51962283 & 100.8070637 \\
\hline Percentage of V0 under 100\$ & $72.72 \%$ & $0.00 \%$ \\
\hline & & \\
\hline
\end{tabular}


Table 13: Initial investment $V_{0}$ in the replication of EDHEC and HFRI indices for both reserve assets for second sub-period (2002-2006).

\begin{tabular}{lcc}
\multicolumn{1}{c}{ Fund } & \multicolumn{2}{c}{$V_{0}$} \\
& Reserve 1 & Reserve 2 \\
\hline EDHEC-Convertible Arbitrage & 99.54307232 & 99.91754606 \\
EDHEC-CTA Global & 99.00591261 & 99.85286557 \\
EDHEC-Distressed Securities & 100.2752537 & 100.645535 \\
EDHEC-Emerging Markets & 100.0757102 & 100.4515871 \\
EDHEC-Equity Market Neutral & 99.85680498 & 99.99383759 \\
EDHEC-Event Driven & 99.87363087 & 100.3162115 \\
EDHEC-Fixed Income Arbitrage & 99.88868645 & 100.0907229 \\
EDHEC-Global Macro & 99.84474995 & 100.2384539 \\
EDHEC-Long/Short Equity & 99.60337666 & 100.1087833 \\
EDHEC-Merger Arbitrage & 99.70200103 & 99.99705359 \\
EDHEC-Relative Value & 99.81967336 & 100.109444 \\
EDHEC-Short Selling & 98.05685558 & 99.04396197 \\
EDHEC-Funds of Funds & 99.74332198 & 100.0559835 \\
\hline Percentage of V Und $_{0}$ under 100\$ & $84.62 \%$ & $38.46 \%$ \\
\hline \hline HFRI Convertible Arbitrage Index & 99.60821174 & 99.93483497 \\
HFRI Distressed Securities Index & 100.2391759 & 100.6380069 \\
HFRI Emerging Markets (Total) & 100.0572944 & 100.8595669 \\
HFRI Equity Hedge Index & 99.58364075 & 100.014334 \\
HFRI Equity Market Neutral Index & 99.66759956 & 99.85722405 \\
HFRI Equity Non-Hedge Index & 99.37314042 & 100.2792862 \\
HFRI Event-Driven Index & 99.80612072 & 100.3402519 \\
HFRI Fixed Income (Total) & 99.95688427 & 100.1391919 \\
HFRI Fixed Income: Arbitrage Index & 100.0072767 & 100.1695353 \\
HFRI Fixed Income: High Yield Index & 100.0771647 & 100.3417642 \\
HFRI FOF: Conservative Index & 99.82149692 & 100.0377755 \\
HFRI FOF: Diversified Index & 99.7547993 & 100.0216789 \\
HFRI FOF: Market Defensive Index & 99.56207483 & 99.97381601 \\
HFRI FOF: Strategic Index & 99.62610152 & 99.96801828 \\
HFRI FOF Composite Index & 99.73892366 & 100.0563079 \\
HFRI FOF Composite Index (Off.) & 99.68519975 & 100.0475484 \\
HFRI Fund Weighted Composite Index & 99.78329249 & 100.2000232 \\
HFRI Macro Index & 99.73199639 & 100.3030235 \\
HFRI Merger Arbitrage Index & 99.66510204 & 100.0050475 \\
HFRI Regulation D Index & 99.47794411 & 100.3049513 \\
HFRI Relative Value Arbitrage Index & 99.94588108 & 100.1510614 \\
HFRI Short Selling Index & 98.37750341 & 99.15058551 \\
\hline Percentage of V0 under 100\$ & $81.81 \%$ & $22.73 \%$ \\
\hline & & \\
\hline
\end{tabular}



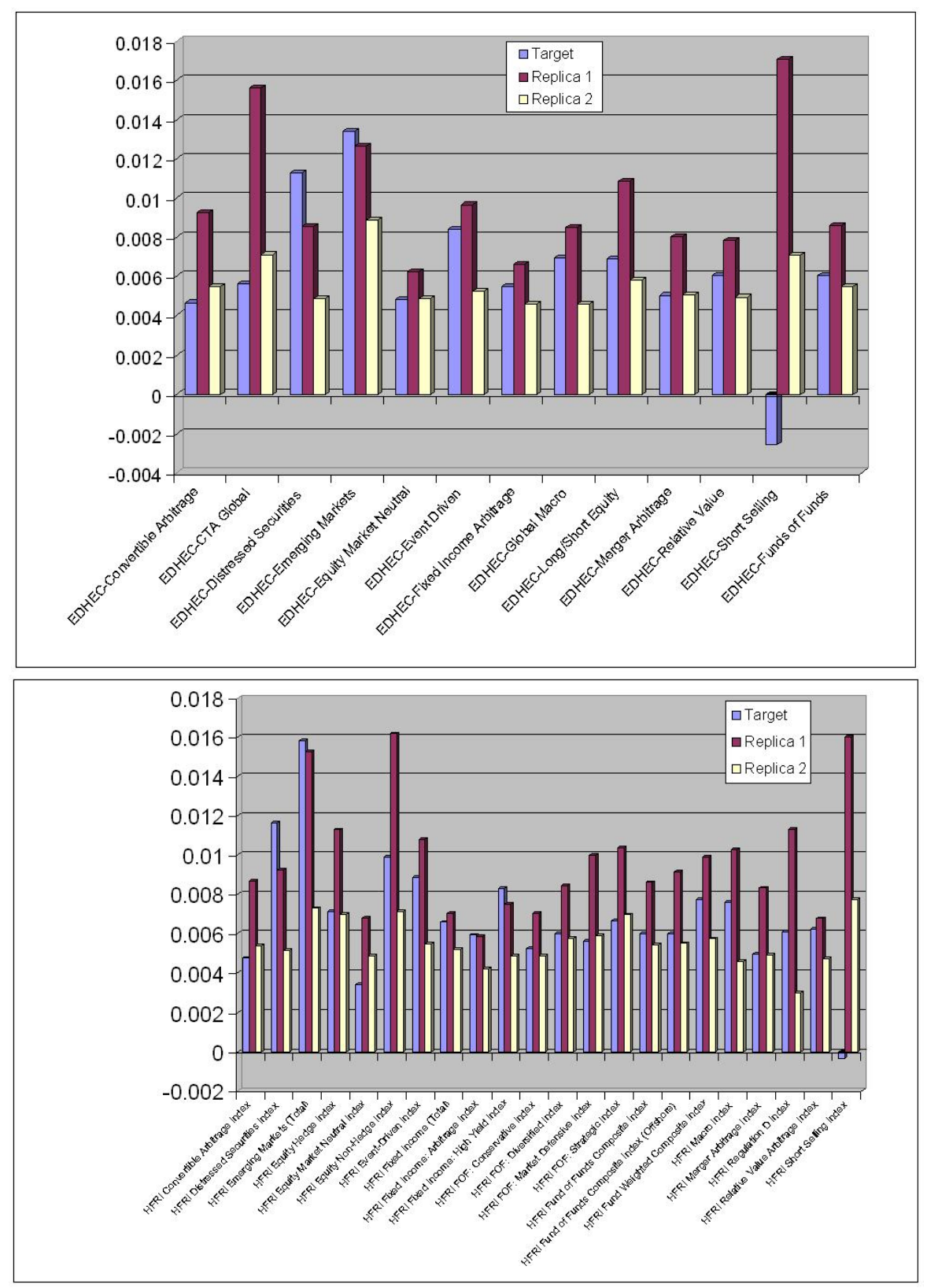

Figure 2: Mean return of replication for both reserve assets vs mean return for EDHEC (top) and HFRI (bottom) indices (2002-2006) 

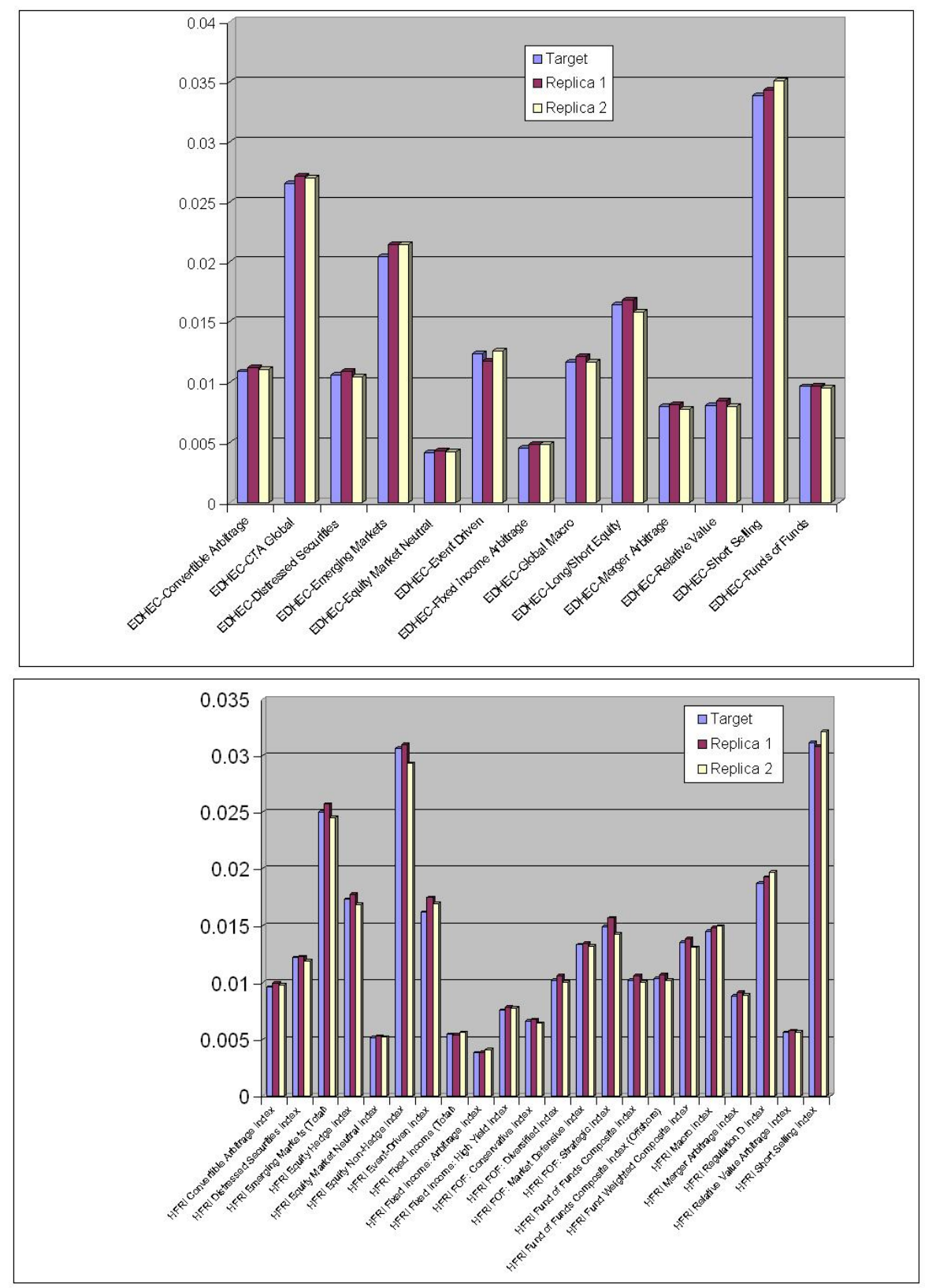

Figure 3: Volatility of the replication with each reserve asset vs target volatility for EDHEC (top) and HFRI (bottom) indices (2002-2006) 

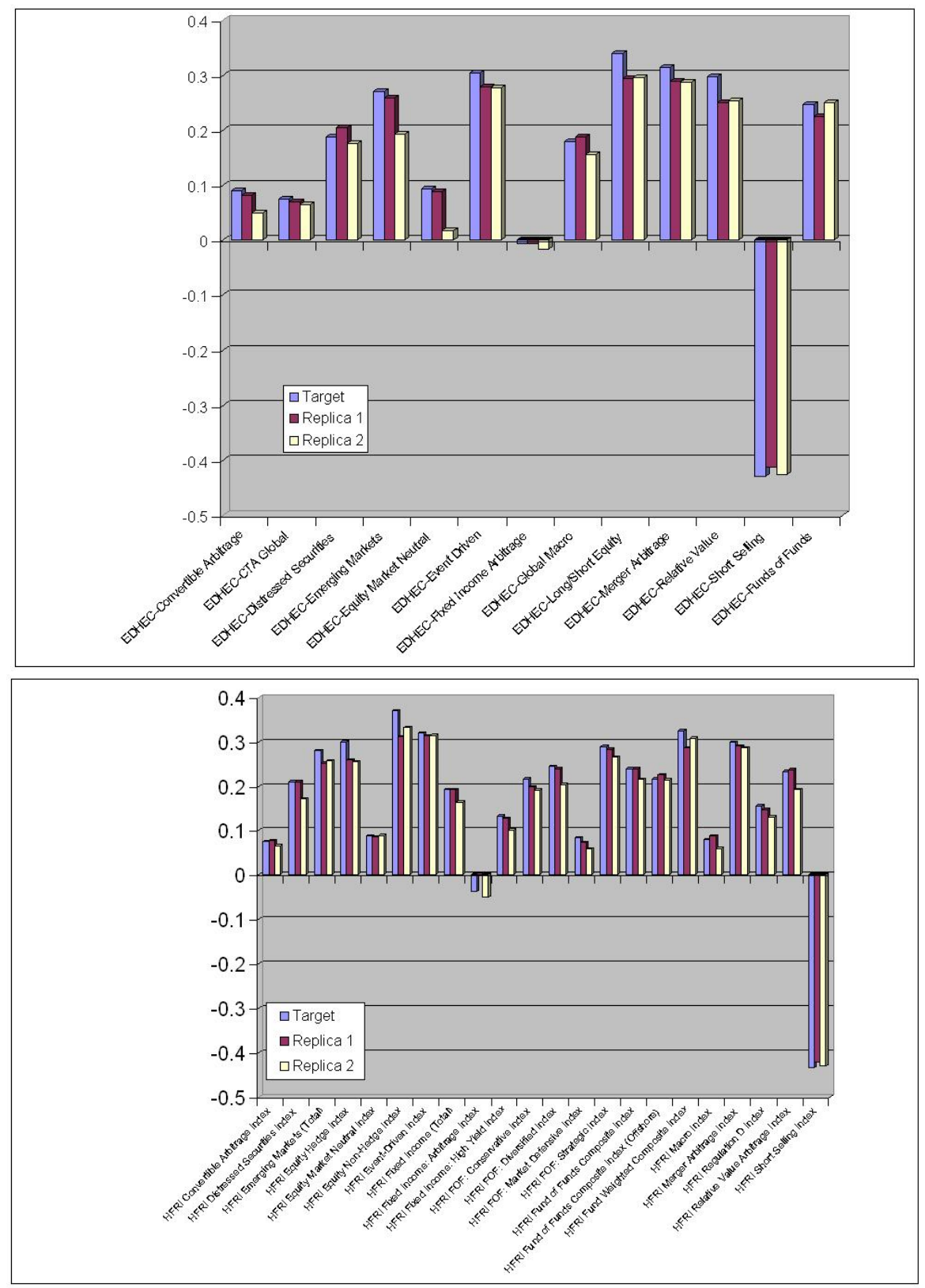

Figure 4: Kendall's tau of the replication with each reserve asset vs target Kendall's tau for EDHEC (top) and HFRI (bottom) indices (2002-2006) 

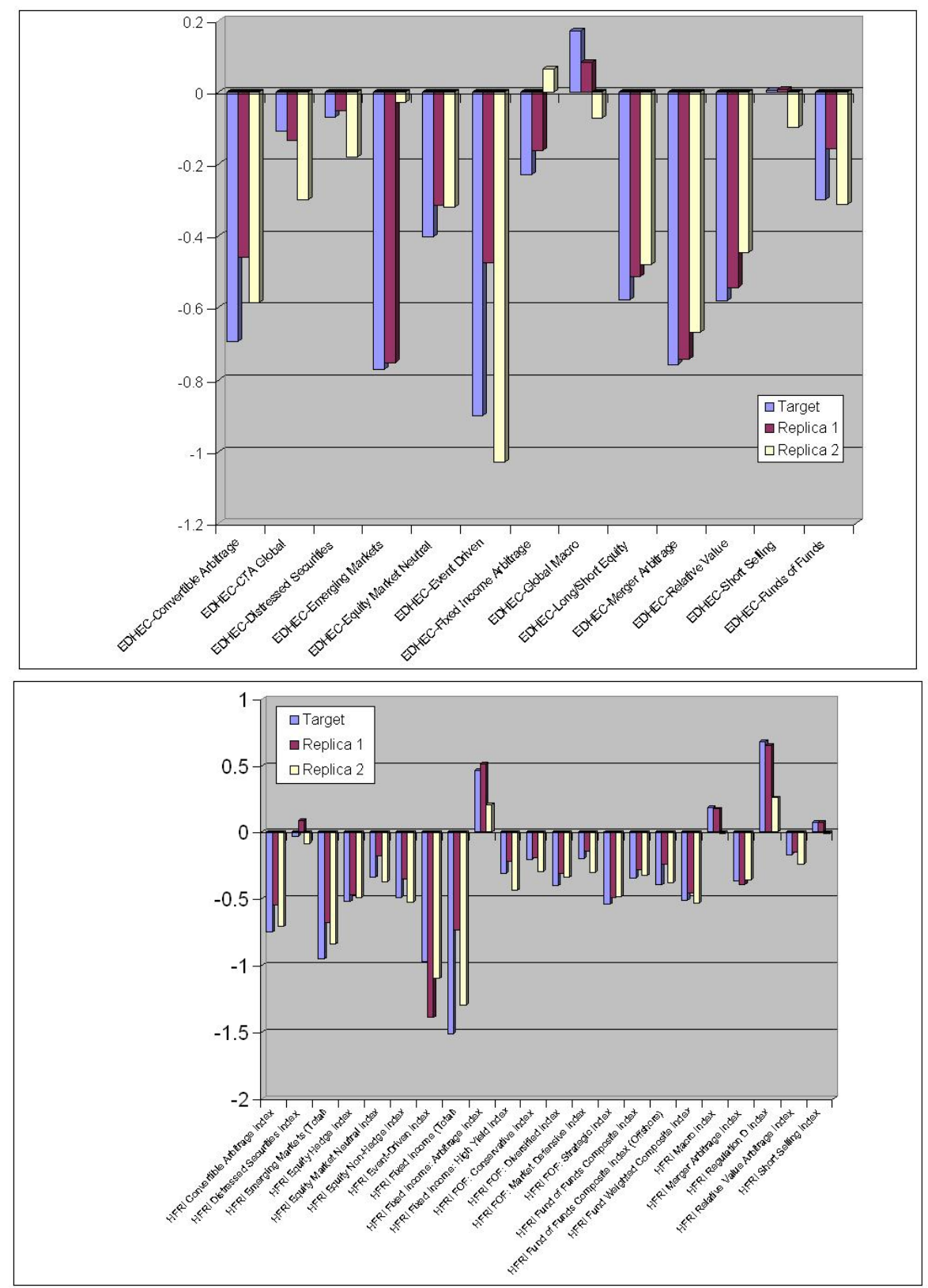

Figure 5: Skewness of the replication with each reserve asset vs target skewness for EDHEC (top) and HFRI (bottom) indices (2002-2006) 

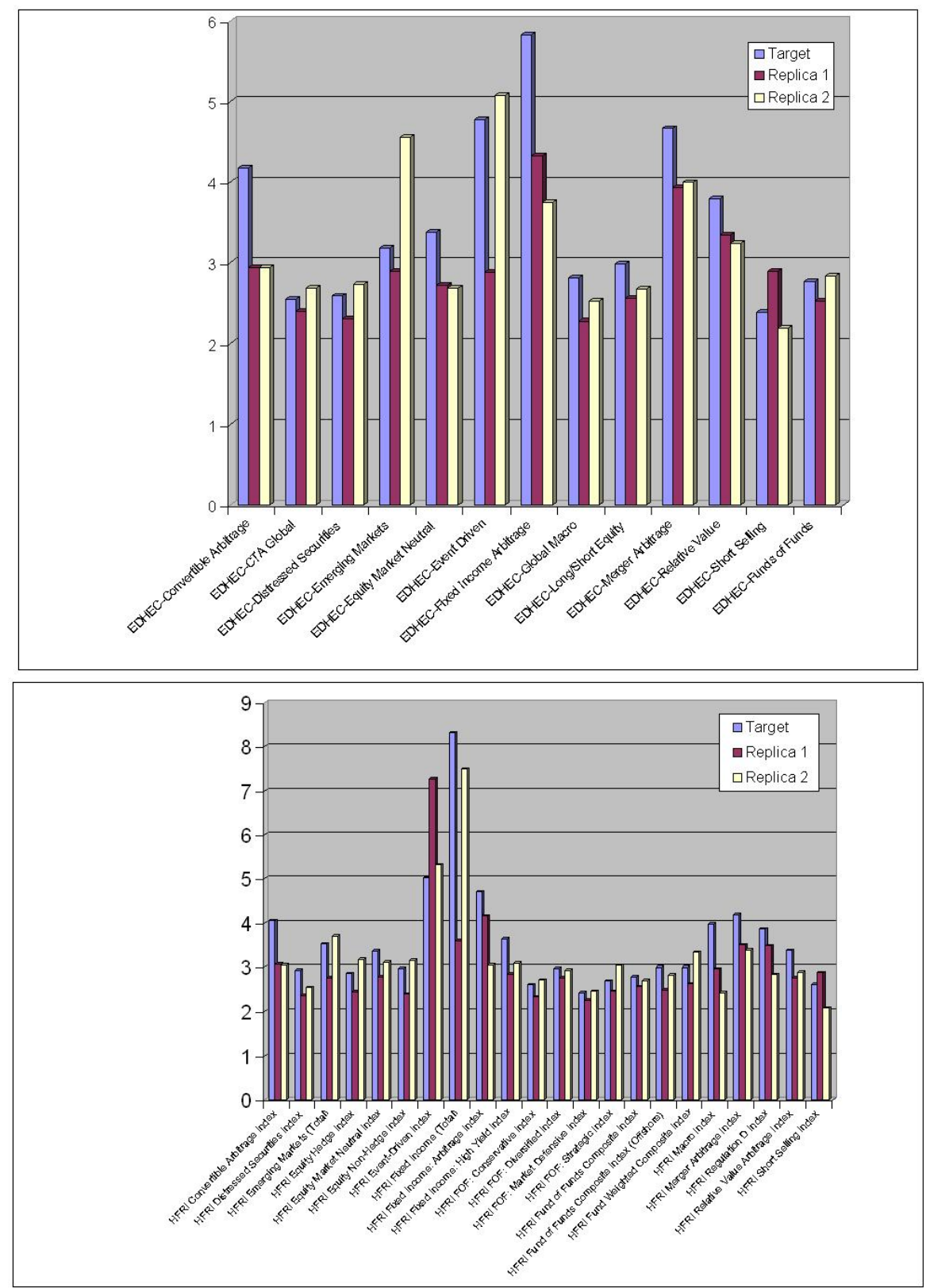

Figure 6: Kurtosis of the replication with each reserve asset vs target kurtosis for EDHEC (top) and HFRI (bottom) indices (2002-2006) 
Table 14: Transaction costs (basis points) of the EDHEC and HFRI indices for each of two reserve assets over the entire period (1997-2006).

\begin{tabular}{|c|c|c|}
\hline \multirow[t]{2}{*}{ Fund } & \multicolumn{2}{|c|}{ Transaction costs } \\
\hline & Reserve 1 & Reserve 2 \\
\hline EDHEC-Convertible Arbitrage & -3.5760 & -2.6937 \\
\hline EDHEC-CTA Global & -5.1209 & -3.6392 \\
\hline EDHEC-Distressed Securities & -3.1461 & -2.9916 \\
\hline EDHEC-Emerging Markets & -10.436 & -8.5692 \\
\hline EDHEC-Equity Market Neutral & -1.1785 & -1.2782 \\
\hline EDHEC-Event Driven & -4.9894 & -3.7833 \\
\hline EDHEC-Fixed Income Arbitrage & -5.6955 & -3.5177 \\
\hline EDHEC-Global Macro & -3.1539 & -3.5487 \\
\hline EDHEC-Long/Short Equity & -3.5405 & -3.5815 \\
\hline EDHEC-Merger Arbitrage & -3.5994 & -2.7794 \\
\hline EDHEC-Relative Value & -2.1994 & -1.8390 \\
\hline EDHEC-Short Selling & -14.472 & -12.690 \\
\hline EDHEC-Funds of Funds & -2.5685 & -2.7680 \\
\hline Average of the transaction costs over the indices & -4.8982 & -4.1292 \\
\hline HFRI Convertible Arbitrage Index & -2.9748 & -2.3503 \\
\hline HFRI Distressed Securities Index & -3.7409 & -3.1175 \\
\hline HFRI Emerging Markets (Total) & -10.409 & -11.231 \\
\hline HFRI Equity Hedge Index & -5.2928 & -5.5529 \\
\hline HFRI Equity Market Neutral Index & -1.9814 & -1.8804 \\
\hline HFRI Equity Non-Hedge Index & -7.6039 & -7.7172 \\
\hline HFRI Event-Driven Index & -3.7228 & -3.3989 \\
\hline HFRI Fixed Income (Total) & -2.8376 & -2.2500 \\
\hline HFRI Fixed Income: Arbitrage Index & -6.1764 & -4.3318 \\
\hline HFRI Fixed Income: High Yield Index & -6.4438 & -3.6841 \\
\hline HFRI FOF: Conservative Index & -2.4110 & -2.1042 \\
\hline HFRI FOF: Diversified Index & -4.7314 & -4.0279 \\
\hline HFRI FOF: Market Defensive Index & -3.6750 & -2.8050 \\
\hline HFRI FOF: Strategic Index & -6.2475 & -6.1420 \\
\hline HFRI FOF Composite Index & -3.7260 & -3.9430 \\
\hline HFRI FOF Composite Index (Off.) & -4.6198 & -4.6972 \\
\hline HFRI Fund Weighted Composite Index & -4.2733 & -4.2082 \\
\hline HFRI Macro Index & -3.3393 & -3.5459 \\
\hline HFRI Merger Arbitrage Index & -3.9681 & -2.8237 \\
\hline HFRI Regulation D Index & -3.5011 & -3.7099 \\
\hline HFRI Relative Value Arbitrage Index & -2.4469 & -1.7284 \\
\hline HFRI Short Selling Index & -19.302 & -17.595 \\
\hline Average of the transaction costs over the indices & -5.1557 & -4.6747 \\
\hline
\end{tabular}


Table 15: Hedging errors (basis per points) of the EDHEC and HFRI indices for each of two reserve assets over the entire period (1997-2006).

\begin{tabular}{|c|c|c|}
\hline \multirow[t]{2}{*}{ Fund } & \multicolumn{2}{|c|}{ Hedging error } \\
\hline & Reserve 1 & Reserve 2 \\
\hline EDHEC-Convertible Arbitrage & -5.022966343 & 2.689724779 \\
\hline EDHEC-CTA Global & -8.058744042 & 5.645421806 \\
\hline EDHEC-Distressed Securities & 4.124754378 & 19.47155871 \\
\hline EDHEC-Emerging Markets & -11.21163859 & 13.259774 \\
\hline EDHEC-Equity Market Neutral & -1.471590683 & 1.56198415 \\
\hline EDHEC-Event Driven & -3.020763751 & 6.22406221 \\
\hline EDHEC-Fixed Income Arbitrage & -5.177575949 & 3.189905767 \\
\hline EDHEC-Global Macro & -4.053867497 & 4.395207 \\
\hline EDHEC-Long/Short Equity & 4.47809413 & 3.734220311 \\
\hline EDHEC-Merger Arbitrage & -3.442046302 & 2.242736202 \\
\hline EDHEC-Relative Value & -1.10554998 & 2.836227619 \\
\hline EDHEC-Short Selling & -24.29013217 & 18.8506452 \\
\hline EDHEC-Funds of Funds & 2.033494462 & 8.749446216 \\
\hline Average of the hedging errors over the indices & -4.324502488 & 7.142377997 \\
\hline HFRI Convertible Arbitrage Index & -4.675913708 & 2.609102503 \\
\hline $\mathrm{HFI}$ & 3.722398591 & 84332 \\
\hline ng Markets (Total) & 7.097564556 & 12.66959323 \\
\hline HFRI Equity Hedge Index & -1.643622346 & 11.19037495 \\
\hline HFRI Equity Market Neutral Index & -2.258515275 & 2.472596466 \\
\hline HFRI Equity Non-Hedge Index & 7.453328183 & 4.603254198 \\
\hline HFRI Event-Driven Index & 2.862294451 & 12.29110626 \\
\hline HFRI Fixed Income (Total) & -2.603139406 & 2.402853856 \\
\hline HFRI Fixed Income: Arbitra & -4.087640896 & 4.977681096 \\
\hline HFRI Fixed Ir & 2.638073684 & 2.387582196 \\
\hline HFRI FOF: C & -2.598299696 & 2.863947585 \\
\hline HFRI FC & -5.7248332 & -2.263005293 \\
\hline HFRI FOF: Mark & -7.19063663 & 3.850690389 \\
\hline HFRI FOF: Stra & -8.584197214 & 7.510126485 \\
\hline HFRI FOF Com & -4.800243375 & 3.856993799 \\
\hline HFRI FOF Coml & -7.540482923 & 5.850515308 \\
\hline HFRI Fund Weighted Composite Index & 2.244013529 & 15.42135204 \\
\hline HFRI Macro Index & -2.491140954 & 7.274298256 \\
\hline HFRI Merger Arbitrage Index & -3.635490602 & 2.457954561 \\
\hline HFRI Regulation D Index & -4.354249204 & 3.999422953 \\
\hline HFRI Relative Value Arbitrage Index & -1.757126584 & 4.245628798 \\
\hline HFRI Short Selling Index & -30.41350326 & 21.98728382 \\
\hline Average of the hedging errors or & -3.106425558 & 6.989782153 \\
\hline
\end{tabular}


Table 16: Hedging errors (basis per points) of the EDHEC and HFRI indices for each of two reserve assets over the first sub-period (1997-2001).

\begin{tabular}{|c|c|c|}
\hline \multirow[t]{2}{*}{ Fund } & \multicolumn{2}{|c|}{ Hedging error } \\
\hline & Reserve 1 & Reserve 2 \\
\hline EDHEC-Convertible Arbitrage & -5.854414114 & 7.331726159 \\
\hline EDHEC-CTA Global & -3.261304874 & 15.48344278 \\
\hline EDHEC-Distressed Securities & -10.63141111 & 18.21688996 \\
\hline EDHEC-Emerging Markets & -41.58467617 & 10.40839934 \\
\hline EDHEC-Equity Market Neutral & 0.216747837 & 4.117171088 \\
\hline EDHEC-Event Driven & 5.530304616 & 15.02572238 \\
\hline EDHEC-Fixed Income Arbitrage & -10.48685482 & 12.72732957 \\
\hline EDHEC-Global Macro & -1.950399253 & 10.81371999 \\
\hline EDHEC-Long/Short Equity & -5.472302407 & 8.63029379 \\
\hline EDHEC-Merger Arbitrage & -7.268360093 & 9.778517204 \\
\hline EDHEC-Relative Value & 12.74567524 & 8.974747668 \\
\hline EDHEC-Short Selling & 9.60796941 & 55.3754198 \\
\hline EDHEC-Funds of Funds & -12.45957574 & 9.552013774 \\
\hline Average of the hedging errors over the indices & -5.4514308 & 14.34118411 \\
\hline HFRI Convertible Arbitrage Index & -4.443351952 & 3.162705469 \\
\hline $\mathrm{HFI}$ & -9.790346341 & 05189 \\
\hline Markets (Total) & -35.23487925 & 15.15013559 \\
\hline HFRI Equity Hedge Index & -15.50411415 & 10.73944888 \\
\hline HFRI Equity Market Neutral Index & -3.470329903 & 4.313327157 \\
\hline HFRI Equity Non-Hedge Index & -23.74524481 & 12.04989301 \\
\hline HFRI Event-Driven Index & -14.22269812 & 2.919363113 \\
\hline HFRI Fixed Income (Total) & -8.573296126 & 5.631050796 \\
\hline HFRI Fixed Income: Arbitra & -4.419486285 & 7.911636813 \\
\hline HFRI Fixed I & -11.13974405 & 8.52434995 \\
\hline HFRI FOF: C & -0.431323396 & 5.769353502 \\
\hline HFRI F & -35.19300862 & 10.4684057 \\
\hline HFRI FOF: Market Def & -10.3549352 & 11.11226975 \\
\hline HFRI F & -12.24470309 & 47099 \\
\hline HFRI FOF Coml & -7.634859635 & 9.191341753 \\
\hline HFRI FOF Com & -9.434687073 & 11.45793373 \\
\hline HFRI Fund Weighted Composite Index & -24.92998374 & 8.097748863 \\
\hline HFRI Macro Index & 3.853207823 & 15.60074834 \\
\hline HFRI Merger Arbitrage Index & -3.887143693 & 5.073019858 \\
\hline HFRI Regulation D Index & -2.431374814 & 13.42772207 \\
\hline HFRI Relative Value Arbitrage Index & -12.36688683 & -0.033279555 \\
\hline HFRI Short Selling Index & -11.04287744 & 4.216592611 \\
\hline Average of the hedging errors or & -11.66554849 & 8.794331377 \\
\hline
\end{tabular}


Table 17: Hedging errors (basis per points) of the EDHEC and HFRI indices for each of two reserve assets over the second sub-period (2002-2006).

\begin{tabular}{|c|c|c|}
\hline \multirow[t]{2}{*}{ Fund } & \multicolumn{2}{|c|}{ Hedging error } \\
\hline & Reserve 1 & Reserve 2 \\
\hline EDHEC-Convertible Arbitrage & -0.216644211 & 14.71418971 \\
\hline EDHEC-CTA Global & -6.582901453 & 36.52509979 \\
\hline EDHEC-Distressed Securities & -1.016305328 & 13.72616412 \\
\hline EDHEC-Emerging Markets & -10.13043018 & 31.53349596 \\
\hline EDHEC-Equity Market Neutral & -1.061827125 & 5.534718588 \\
\hline EDHEC-Event Driven & 6.84618159 & 12.12860342 \\
\hline EDHEC-Fixed Income Arbitrage & -0.469146864 & 9.667485841 \\
\hline EDHEC-Global Macro & -0.423280278 & 15.97182143 \\
\hline EDHEC-Long/Short Equity & -4.781157282 & 11.93987295 \\
\hline EDHEC-Merger Arbitrage & 2.432702745 & 11.46724918 \\
\hline EDHEC-Relative Value & 1.392743596 & 6.816342792 \\
\hline EDHEC-Sho & 8.422391583 & 44.59416583 \\
\hline EDHEC-Funds of Funds & -0.055444422 & 12.87295253 \\
\hline Average of the hedging errors over th & -0.434085972 & 17.49939709 \\
\hline HFRI Convertible Arbitrage Index & 326757 & 7712 \\
\hline Securities Index & 0.409148738 & 14.75409472 \\
\hline Iarkets (Total) & -9.733473043 & 18.61313588 \\
\hline HFRI Equity Hedge Index & -7.44974449 & 20.13641716 \\
\hline Market Neutral Index & -0.864838511 & 7.778622358 \\
\hline HFRI Equity Non-Hedge Index & -12.1917044 & 22.30909531 \\
\hline Oriven Index & 2.282364634 & 16.12105281 \\
\hline Income (Total) & 0.028468682 & 4.514146651 \\
\hline me: Arbitrage 1 & -0.000830608 & 8.164211829 \\
\hline d Income: High & 0.197970856 & 10.94636336 \\
\hline F: Conservative Index & -3.168935239 & 6.353466396 \\
\hline HFRI FOF: Diversified Index & -0.756117028 & 52293 \\
\hline Defensive Index & -4.356542614 & 39458 \\
\hline HFRI FOF: Stra & -5.764923859 & 28265 \\
\hline HFRI FOF Composite Index & -0.639004576 & 50198 \\
\hline HFRI FOF Composite Index (Off.) & -0.945047981 & 9.668544712 \\
\hline HFRI Fund Weighted Composite Index & -4.808300645 & 13.62398725 \\
\hline HFRI Macro Index & 1.031221433 & 30.93949304 \\
\hline HFRI Merger Arbitrage Index & 2.456653636 & 12.85418557 \\
\hline HFRI Regulation D Index & 0.291293742 & 28.74394205 \\
\hline HFRI Relative Value Arbitrage In & 0.400473588 & 7.062009818 \\
\hline HFRI Short Selling Index & 7.951857962 & 38.58089671 \\
\hline Average of the hedging errors over the $i$ & -1.607621953 & 15.30325292 \\
\hline
\end{tabular}




\section{A Estimation and goodness-of-fit}

In this section, we describe the estimation procedure and the goodness-of-fit tests.

\section{A.1 EM algorithm for bivariate Gaussian mixtures}

Let $y_{1}, \ldots, y_{n}$ be a random sample from a bivariate Gaussian mixture with parameters $\pi=$ $\left(\pi_{k}\right)_{k=1}^{m}, \mu=\left(\mu_{k}\right)_{k=1}^{m}$ and $A=\left(A_{k}\right)_{k=1}^{m}$. Start with an initial estimator $\theta^{(0)}$. Given an estimator $\theta^{(\ell)}=\left(\pi^{(\ell)}, \mu^{(\ell)}, A^{(\ell)}\right)$ of the parameters $\theta=(\pi, \mu, A)$, set

$$
\pi_{k}\left(y_{i}, \theta^{(\ell)}\right)=\frac{\pi_{k}^{(\ell)} \phi_{2}\left(y_{i} ; \mu_{k}^{(\ell)}, A_{k}^{(\ell)}\right)}{\sum_{j=1}^{m} \pi_{j}^{(\ell)} \phi_{2}\left(y_{i} ; \mu_{j}^{(\ell)}, A_{j}^{(\ell)}\right)}, \quad i=1, \ldots, n,
$$

and define the new estimator $\theta^{(\ell+1)}=\left(\pi^{(\ell+1)}, \mu^{(\ell+1)}, A^{(\ell+1)}\right)$ viz.

$$
\begin{gathered}
\pi_{k}^{(\ell+1)}=\frac{1}{n} \sum_{i=1}^{n} \pi_{k}\left(y_{i}, \theta^{(\ell)}\right), \\
\mu_{k}^{(\ell+1)}=\frac{1}{n} \sum_{i=1}^{n} y_{i} \pi_{k}\left(y_{i}, \theta^{(\ell)}\right) / \pi_{k}^{(\ell+1)},
\end{gathered}
$$

and

$$
A_{k}^{(\ell+1)}=\frac{1}{n} \sum_{i=1}^{n}\left(y_{i}-\mu_{k}^{(\ell+1)}\right)\left(y_{i}-\mu_{k}^{(\ell+1)}\right)^{\top} \pi_{k}\left(y_{i}, \theta^{(\ell)}\right) / \pi_{k}^{(\ell+1)}
$$

for $k=1, \ldots, m$. As $\ell$ increases, the numbers $\left\{\pi_{k}\left(y_{i}, \theta^{(\ell)}\right) ; k=1, \ldots, i=1, \ldots, n\right\}$ stabilize and the estimators converge.

\section{A.2 Tests of goodness-of-fit}

Testing goodness-of-fit is an essential step for modelling data. There are many tests available but to our knowledge, the best ones are based on empirical processes (Genest and Rémillard, 2005, Genest et al., 2007). Here, we only consider two tests based on the so-called Rosenblatt's transform. The first one is due to Durbin (1973) but the calculation of $P$-values is recent (Stute et al., 1993). For the second test designed for testing goodness-of-fit for bivariate data, the validity of the algorithm for calculating $P$-values follows from Genest and Rémillard (2005). 


\section{A.3 Tests of goodness-of-fit for a univariate parametric distribu- tion}

Let $X_{1}, \ldots, X_{n}$ be a sample of size $n$ from a (continuous) distribution $F$ on $\mathbb{R}$. Suppose that the hypotheses to be tested are

$$
\mathcal{H}_{0}: F \in \mathcal{F}=\left\{F_{\theta} ; \theta \in \Theta\right\} \quad \text { vs } \quad \mathcal{H}_{1}: F \notin \mathcal{F}
$$

For example, the parametric family $\mathcal{F}$ could be the family of univariate Gaussian mixtures with $m$ regimes.

The proposed test statistic is based on Durbin (1973). Let $\theta_{n}=T_{n}\left(X_{1}, \ldots, X_{n}\right)$ be a regular estimator of $\theta$, in the sense of Genest and Rémillard (2005) and set

$$
D_{n}(u)=\frac{1}{n} \sum_{i=1}^{n} \mathbb{I}\left(U_{i} \leq u\right), \quad u \in[0,1],
$$

where $U_{i}=F_{\theta_{n}}\left(X_{i}\right), i=1, \ldots, n$. To test $\mathcal{H}_{0}$ against $\mathcal{H}_{1}$, one may use the Cramér-von Mises type statistic

$$
\begin{aligned}
S_{n} & =n \int_{0}^{1}\left\{D_{n}(u)-u\right\}^{2} d u \\
& =\frac{1}{n} \sum_{i=1}^{n} \sum_{j=1}^{n}\left\{\frac{U_{i}^{2}+U_{j}^{2}-2 \max \left(U_{i}, U_{j}\right)}{2}+\frac{1}{3}\right\} .
\end{aligned}
$$

Since the $U_{i}$ 's are "almost uniformly distributed on $[0,1]$ " under the null hypothesis, large values of $S_{n}$ should lead to rejection of the null hypothesis. However, in general the limiting distribution of $S_{n}$ depend on the unknown parameter $\theta$. To calculate the $P$-value of $S_{n}$, one can use a parametric bootstrap approach as described below.

a) Calculate $\theta_{n}$ and $S_{n}$.

b) For some large integer $N$ (say 1000), repeat the following steps for every $k \in\{1, \ldots, N\}$ :

(i) Generate a random sample $X_{1, k}, \ldots, X_{n, k}$ from distribution $F_{\theta_{n}}$.

(ii) Calculate

$$
\begin{aligned}
\theta_{n, k} & =T_{n}\left(X_{1, k}, \ldots, X_{n, k}\right), \\
U_{i, k} & =F_{\theta_{n, k}}\left(X_{i, k}\right), \quad i=1, \ldots, n, \\
S_{n, k} & =\frac{1}{n} \sum_{i=1}^{n} \sum_{j=1}^{n}\left\{\frac{U_{i, k}^{2}+U_{j, k}^{2}-2 \max \left(U_{i, k}, U_{j, k}\right)}{2}+\frac{1}{3}\right\} .
\end{aligned}
$$


An approximate $P$-value for the test based on the Cramér-von Mises statistic $S_{n}$ is then given by

$$
\frac{1}{N} \sum_{k=1}^{N} \mathbb{I}\left(S_{n, k}>S_{n}\right) .
$$

\section{A.4 Tests of goodness-of-fit for a bivariate parametric distribution}

Let $\left(X_{1}, Y_{1}\right) \ldots,\left(X_{n}, Y_{n}\right)$ be a sample of size $n$ from a (continuous) distribution $F$ on $\mathbb{R}^{2}$. Suppose that the hypotheses to be tested are

$$
\mathcal{H}_{0}: F \in \mathcal{F}=\left\{F_{\theta} ; \theta \in \Theta\right\} \quad \text { vs } \quad \mathcal{H}_{1}: F \notin \mathcal{F}
$$

For example, the parametric family $\mathcal{F}$ could be the family of bivariate Gaussian mixtures with $m$ regimes. Denote by $G_{\theta}$ the distribution function of $X_{i}$ and let $H_{\theta}$ be the conditional distribution function of $Y_{i}$ given $X_{i}$, i.e., $H_{\theta}(x, y)=P\left(Y_{i} \leq y \mid X_{i}=x\right)$.

The proposed test statistic is based on Durbin (1973) and the Rosenblatt's transform (Rosenblatt, 1952).

Suppose that $\theta_{n}=T_{n}\left(X_{1}, Y_{1}, \ldots, X_{n}, Y_{n}\right)$ is a regular estimator of $\theta$, in the sense of Genest and Rémillard (2005) and set

$$
D_{n}(u, v)=\frac{1}{n} \sum_{i=1}^{n} \mathbb{I}\left(U_{i} \leq u, V_{i} \leq v\right), \quad u, v \in[0,1],
$$

where $U_{i}=G_{\theta_{n}}\left(X_{i}\right), V_{i}=H_{\theta_{n}}\left(X_{i}, Y_{i}\right), i=1, \ldots, n$. To test $\mathcal{H}_{0}$ against $\mathcal{H}_{1}$, one may use the Cramér-von Mises type statistic

$$
\begin{aligned}
& S_{n}= n \int_{0}^{1} \int_{0}^{1}\left\{D_{n}(u, v)-u v\right\}^{2} d u d v \\
&=\frac{1}{n} \sum_{i=1}^{n} \sum_{j=1}^{n}\left[\frac{1}{9}-\frac{1}{4}\left(1-U_{i}^{2}\right)\left(1-V_{i}^{2}\right)-\frac{1}{4}\left(1-U_{j}^{2}\right)\left(1-V_{j}^{2}\right)\right. \\
&\left.\quad+\left\{1-\max \left(U_{i}, U_{j}\right)\right\}\left\{1-\max \left(V_{i}, V_{j}\right)\right\}\right] .
\end{aligned}
$$

Since the pairs $\left(U_{i}, V_{i}\right)$ 's are "almost uniformly distributed on $[0,1]^{2}$ " under the null hypothesis, large values of $S_{n}$ should lead to rejection of the null hypothesis. However, in general the limiting distribution of $S_{n}$ depend on the unknown parameter $\theta$. To calculate the $P$-value of $S_{n}$, one can use a parametric bootstrap approach as described below.

a) Calculate $\theta_{n}$ and $S_{n}$. 
b) For some large integer $N$ (say 1000), repeat the following steps for every $k \in\{1, \ldots, N\}$ :

(i) Generate a random sample $\left(X_{1, k}, Y_{1, k}\right), \ldots,\left(X_{n, k}, Y_{n, k}\right)$ from distribution $F_{\theta_{n}}$.

(ii) Calculate

$$
\begin{aligned}
\theta_{n, k}^{*}= & T_{n}\left(X_{1, k}, Y_{1, k}, \ldots, X_{n, k}, Y_{n, k}\right), \\
U_{i, k}= & G_{\theta_{n, k}}\left(X_{i, k}\right), \quad V_{i, k}=H_{\theta_{n, k}}\left(X_{i, k}, Y_{i, k}\right), \quad i=1, \ldots, n \\
S_{n, k}= & \frac{1}{n} \sum_{i=1}^{n} \sum_{j=1}^{n}\left[\frac{1}{9}-\frac{1}{4}\left(1-U_{i, k}^{2}\right)\left(1-V_{i, k}^{2}\right)-\frac{1}{4}\left(1-U_{j, k}^{2}\right)\left(1-V_{j, k}^{2}\right)\right. \\
& \left.+\left\{1-\max \left(U_{i, k}, U_{j, k}\right)\right\}\left\{1-\max \left(V_{i, k}, V_{j, k}\right)\right\}\right] .
\end{aligned}
$$

An approximate $P$-value for the test based on the Cramér-von Mises statistic $S_{n}$ is then given by

$$
\frac{1}{N} \sum_{k=1}^{N} \mathbb{I}\left(S_{n, k}>S_{n}\right) .
$$

\section{B Implementation of the dynamic trading strategy}

Before describing the algorithm, it is important to define what is meant by a partition. Here we assume that $S_{t}=\omega_{t}\left(S_{t-1}, \xi_{t}\right), \xi_{t} \sim \mu_{t}$ being independent of $\mathcal{F}_{t-1}, t=1, \ldots, T$.

Definition B.1 A partition $\mathcal{P}$ of a compact convex set $K$, is any finite set $\mathcal{P}=\left\{S_{1}, \ldots, S_{m}\right\}$ of simplexes with disjoint non empty interiors, so that $K=\bigcup_{j=1}^{m} S_{j}$. The set of vertices of the partition $\mathcal{P}$ is denoted by $\mathcal{V}(\mathcal{P})$.

Note that $K$ is then the convex hull generated by $\mathcal{V}(\mathcal{P})$.

The algorithm is based on Monte Carlo simulations, combined with a sequence of approximations on compact sets $K_{0}, \ldots, K_{T-1}$, determined by partitions $\mathcal{P}_{0}, \ldots, \mathcal{P}_{T-1}$. The idea behind the algorithm is quite simple: Given approximations $\tilde{f}_{t}$, of $f_{t}$, one first get $\hat{L}_{1 t}, \hat{L}_{2 t}, \hat{A}_{t}, \hat{\Delta}_{t}, \hat{U}_{t}$ and $\hat{f}_{t-1}$, by estimating these functions at every vertices $x \in \mathcal{V}\left(\mathcal{P}_{t-1}\right)$, using Monte Carlo simulations, and then, one uses a linear interpolation to extend them at any point $x \in K_{t-1}$. More precisely, one may proceed through the following steps.

\section{B.1 Algorithm}

- Set $\tilde{f}_{T}=f_{T}$; 
- For each $t=T, \ldots, 1$

- Generate $\xi_{1, t}, \ldots, \xi_{N_{t}, t}$ according to $\mu_{t}$;

- For every $s \in \mathcal{V}\left(\mathcal{P}_{t-1}\right)$, calculate

$$
\begin{aligned}
\hat{L}_{1 t}(s) & =\frac{1}{N_{t}} \sum_{i=1}^{N_{t}} \omega_{t}\left(s, \xi_{i, t}\right) \\
\hat{L}_{2 t}(s) & =\frac{1}{N_{t}} \sum_{i=1}^{N_{t}} \omega_{t}\left(s, \xi_{i, t}\right) \omega_{t}\left(s, \xi_{i, t}\right)^{\top} \\
\hat{A}_{t}(s) & =\hat{L}_{2 t}(s)-\hat{L}_{1 t}(s) \hat{L}_{1 t}(s)^{\top} \\
\hat{\psi}_{t}(s) & =\hat{A}_{t}(s)^{-1} \frac{1}{N_{t}} \sum_{i=1}^{N_{t}}\left\{\omega_{t}\left(s, \xi_{i, t}\right)-\hat{L}_{1 t}(s)\right\} \tilde{f}_{t}\left\{\omega_{t}\left(s, \xi_{i, t}\right)\right\} \\
\hat{U}_{t}(s, x) & =1-\left\{\hat{L}_{1 t}(s)-\beta_{t-1} s / \beta_{t}\right\}^{\top} \hat{A}_{t}(s)^{-1}\left\{x-\hat{L}_{1 t}(s)\right\} \\
\hat{f}_{t-1}(s) & =\frac{\beta_{t}}{\beta_{t-1}} \frac{1}{N_{t}} \sum_{i=1}^{N_{t}} \hat{U}_{t}\left\{s, \omega_{t}\left(s, \xi_{i, t}\right)\right\} \tilde{f}_{t}\left\{\omega_{t}\left(s, \xi_{i, t}\right)\right\} .
\end{aligned}
$$

- Interpolate linearly $\hat{\Delta}_{t}$ and $\hat{f}_{t-1}$ over $K_{t-1}$ and extend it to all of $\mathfrak{X}$.

A detailed description of the linear interpolation implementation techniques is given below, but first, the following result adapted from Del Moral et al. (2006), confirms that the algorithm produces good approximations.

Theorem 2 Suppose that $f_{T}$ is continuous and that for all $1 \leq t \leq T, \omega_{t}(\cdot, \xi)$ are continuous for a fixed $\xi$. Let $K_{0}$ be a given compact convex subset of $\mathfrak{X}$. Let $\epsilon>0$ be given. Then one can find compact convex sets $K_{1}, \ldots, K_{n-1} \subset \mathfrak{X}$, partitions $\mathcal{P}_{0}, \ldots \mathcal{P}_{n-1}$ generating respectively $K_{0}, \ldots, K_{n-1}$, and integers $N_{10}, \ldots, N_{n 0}$, so that for the simple interpolation method,

$$
\max _{1 \leq k \leq n}\left\|\psi_{t}-\tilde{\psi}_{t}\right\|_{K_{t-1}}<\epsilon
$$

and

$$
\max _{0 \leq k \leq n-1}\left\|f_{t}-\tilde{f}_{t}\right\|_{K_{t}}<\epsilon
$$

whenever $N_{1} \geq N_{10}, \ldots, N_{n} \geq N_{n 0}$.

\section{B.2 Linear interpolations}

Definition B.2 Given a function $h$ and a partition $\mathcal{P}$ of $K$, a linear interpolation of $h$ over $\mathcal{P}$ is the (unique) function $\tilde{g}$ defined in the following way: 
If $S \in \mathcal{P}$ is a simplex with vertices $x_{1}, \ldots, x_{d+1}$, then set

$$
\tilde{h}(x)=\sum_{i=1}^{d+1} \lambda_{i} h\left(x_{i}\right)
$$

where the barycenters $\left\{\lambda_{1}, \ldots, \lambda_{d+1}\right\}$ are the unique solution of

$$
x=\sum_{i=1}^{d+1} \lambda_{i} x_{i}, \quad \sum_{i=1}^{d+1} \lambda_{i}=1, \quad \lambda_{i} \in[0,1], i=1, \ldots d+1 .
$$

If $x \notin K$, let $x_{K}$ be the (unique) closest point to $x$ that belongs to $K$, and set $\tilde{h}(x)=\tilde{h}\left(x_{K}\right)$. Uniqueness follows from the convexity of $K$ and the strict convexity of the Euclidean norm.

Remark B.1 Note that since each $x_{i}$ is extreme in $S$, the unique solution of

$$
x_{i}=\sum_{j=1}^{d+1} \lambda_{j} x_{j}, \quad \sum_{j=1}^{d+1} \lambda_{j}=1, \quad \lambda_{j} \in[0,1], j=1, \ldots d+1,
$$

is $\lambda_{i}=1$ and $\lambda_{j}=0$ for all $j \neq i$, yielding $\tilde{g}\left(x_{i}\right)=g\left(x_{i}\right)$ for all $1 \leq i \leq m$. Moreover, $\tilde{g}$ is affine on each simplex, justifying the term "linear interpolation".

Finally, $\tilde{g}$ is continuous and bounded on $\mathfrak{X}$ and

$$
\sup _{x \in K}|g(x)-\tilde{g}(x)| \leq \omega(g, K, \operatorname{mesh}(\mathcal{P}))
$$

where

$$
\operatorname{mesh}(\mathcal{P})=\max _{\mathrm{S} \in \mathcal{P}} \sup _{\mathrm{x}, \mathrm{z} \in \mathrm{S}}\|\mathrm{x}-\mathrm{z}\|
$$

and $\omega(g, K, \delta)$ is the modulus of continuity of $g$ over $K$, i.e.

$$
\omega(g, K, \delta)=\sup _{x, z \in K,\|x-z\| \leq \delta}|g(x)-g(z)| .
$$

Example B.1 Suppose $d=1$. Then the linear interpolation $\tilde{g}$ of a monotone (respectively convex) function $g$ on $K=[a, b]$ is monotone (respectively convex). To see that, set $a_{i}=$ $a+i(b-a) / m, i=0, \ldots, m$ and let $\mathcal{P}$ be the partition given by $\mathcal{P}=\left\{\left[a_{i-1}, a_{i}\right] ; i=1, \ldots, m\right\}$. Set $\Delta_{i}=\frac{g\left(a_{i}\right)-g\left(a_{i-1}\right)}{a_{i}-a_{i-1}}, 1 \leq i \leq m$. Then the linear interpolation of $g$ over $K$ is given by

$$
\tilde{h}(x)= \begin{cases}h(a), & x \leq a, \\ h\left(a_{i}\right)+\left(x-a_{i}\right) \Delta_{i+1}, & x \in\left[a_{i}, a_{i+1}\right], i=0, \ldots, m-1, \\ h(b) & x \geq b .\end{cases}
$$

If $h$ is monotone, the slopes $\Delta_{i}$ all have the same sign, so $\tilde{h}$ has the same monotonicity. If $h$ is convex, the slopes $\Delta_{i}$ are non decreasing, so $\tilde{h}$ is also convex. 
Example B.2 Suppose $d=2$. First define interpolation on $[0,1]^{2}$. Suppose that $h$ is known at points $(0,0),(0,1),(1,0)$ and $(1,1)$. If one wants to linearly interpolate $h$, as in Definition B.Q, a convenient choice for the partition $\mathcal{P}$ of $[0,1]^{2}$ is $\mathcal{P}=\left\{S_{1}, S_{2}\right\}$ where

$$
S_{1}=\left\{\left(x_{1}, x_{2}\right) \in[0,1]^{2} ; x_{1} \leq x_{2}\right\} \quad S_{1}=\left\{\left(x_{1}, x_{2}\right) \in[0,1]^{2} ; x_{1} \geq x_{2}\right\}
$$

Any $x \in S_{1}$ can be uniquely written as

$$
x=\lambda_{1}(0,1)+\lambda_{2}(1,1)+\lambda_{3}(0,0),
$$

with $\lambda_{2}=x_{1}, \lambda_{1}=x_{2}-x_{1}$, and $\lambda_{3}=1-x_{2}$, so one can define

$$
\begin{aligned}
\tilde{h}(x) & =\lambda_{1} h(0,1)+\lambda_{2} h(1,1)+\lambda_{3} h(0,0) \\
& =h(0,0)+x_{1}\{h(1,1)-h(0,1)\}+x_{2}\{h(0,1)-h(0,0)\} .
\end{aligned}
$$

Similarly, for any $x \in S_{2}$, one obtains

$$
\begin{aligned}
\tilde{h}(x) & =\lambda_{1} h(0,1)+\lambda_{2} h(1,1)+\lambda_{3} h(0,0) \\
& =h(0,0)+x_{1}\{h(1,0)-h(0,0)\}+x_{2}\{h(1,1)-h(1,0)\} .
\end{aligned}
$$

Suppose now that $K=\left[a_{1}, b_{1}\right] \times\left[a_{2}, b_{2}\right]$ is partition into smaller rectangles. On each of these sub-rectangles $R=\left[y_{1}, y_{2}\right] \times\left[z_{1}, z_{2}\right]$, just use the linear interpolation on $[0,1]^{2}$ by transforming $x \in R$ into $x^{\prime}=\left(x_{1}^{\prime}, x_{2}^{\prime}\right) \in[0,1]^{2}$ through the mapping $x_{1}^{\prime}=\frac{x_{1}-y_{1}}{y_{2}-y_{1}}, x_{2}^{\prime}=\frac{x_{2}-z_{1}}{z_{2}-z_{1}}$.

Outside $K, \tilde{h}$ is defined as follows:

$$
\tilde{h}(x)=\left\{\begin{array}{ll}
\tilde{h}\left(x_{1}, a_{2}\right) & \text { if } x \in\left[a_{1}, b_{1}\right] \times\left(-\infty, a_{2}\right) \\
\tilde{h}\left(x_{1}, b_{2}\right) & \text { if } x \in\left[a_{1}, b_{1}\right] \times\left(b_{2}, \infty\right) \\
\tilde{h}\left(a_{1}, x_{2}\right) & \text { if } x \in\left(-\infty, a_{1}\right) \times\left[a_{2}, b_{2}\right] \\
\tilde{h}\left(b_{1}, x_{2}\right) & \text { if } x \in\left(b_{1}, \infty\right) \times\left[a_{2}, b_{2}\right] \\
\tilde{h}\left(a_{1}, a_{2}\right) & \text { if } x \in\left(-\infty, a_{1}\right) \times\left(-\infty, a_{2}\right) \\
\tilde{h}\left(b_{1}, a_{2}\right) & \text { if } x \in\left(b_{1}, \infty\right) \times\left(-\infty, a_{2}\right) \\
\tilde{h}\left(a_{1}, b_{2}\right) & \text { if } x \in\left(-\infty, a_{1}\right) \times\left(b_{2}, \infty\right) \\
\tilde{h}\left(b_{1}, b_{2}\right) & \text { if } x \in\left(b_{1}, \infty\right) \times\left(b_{2}, \infty\right)
\end{array} .\right.
$$

\section{Auxiliary results}

Throughout this appendix, $L^{2}=L^{2}(\Omega, \mathcal{F}, P)$ is the set of all random variables on $(\Omega, \mathcal{F})$ which are square integrable. 
Proposition 1 Suppose that $X$ is non negative random variable on $(\Omega, \mathcal{F}, P)$ such that $E(X)<\infty$. Suppose $\mathcal{G}$ is a sub $\sigma$-algebra of $\mathcal{F}$ and let $Z=E(X \mid \mathcal{G}) \geq 0$, $P$ almost surely. Then for any non negative $\mathcal{G}$-measurable random variable $\xi$, the following equality holds

$$
E(\xi X)=E(\xi Z) .
$$

Proof In the case of bounded random variable $\xi$, the result follows from the very definition of the conditional expectation. In particular it is true for $\xi_{n}=\min (n, \xi) \geq 0$, for any $n \geq 1$. Since $\xi_{n} \uparrow \xi$, it follows from Beppo-Levy theorem that

$$
E(\xi X)=\lim _{n \rightarrow \infty} E\left(\xi_{n} X\right)=\lim _{n \rightarrow \infty} E\left(\xi_{n} Z\right)=E(\xi Z) .
$$

Proposition 2 Suppose that $\xi \in \mathbb{R}^{d}$ and $\eta \in \mathbb{R}$ are $L^{2}$ random variables in $(\Omega, \mathcal{F})$ and suppose that $A=E\left(\xi \xi^{\top} \mid \mathcal{G}\right)$ is invertible, where $\mathcal{G}$ is a sub $\sigma$-algebra of $\mathcal{F}$. Then $\varphi \in \mathbb{R}^{d}$ minimizes $E\left\{\left(\varphi^{\top} \xi-\eta\right)^{2}\right\}$ over all $\varphi \in \mathcal{G}$ such that $\varphi^{\top} \xi \in L^{2}$ if and only if $\varphi=A^{-1} b$, where $b=E(\xi \eta \mid \mathcal{G})$. In particular $\varphi^{\top} \xi$ is square integrable.

Proof Set $\varphi=A^{-1} b$. To prove that $\varphi^{\top} \xi \in L^{2}$, note that it follows from Proposition 1 that

$$
\begin{aligned}
E\left\{\left(\varphi^{\top} \xi\right)^{2}\right\} & =\sum_{i=1}^{d} E\left(\varphi_{i}^{2} \xi_{i}^{2}\right) \\
& =\sum_{i=1}^{d} E\left\{\varphi_{i}^{2} E\left(\xi_{i}^{2} \mid \mathcal{G}\right)\right\} \\
& =\sum_{i=1}^{d} E\left(\varphi_{i}^{2} A_{i i}\right) \\
& =E\left(b^{\top} A^{-1} b\right) .
\end{aligned}
$$

Since $A$ is symmetric and positive definite, there exist a $d \times d$ matrix $M \in \mathcal{G}$ such that $M^{-1}=M^{\top}$ and a $d \times d$ diagonal matrix $\Delta \in \mathcal{G}$ such that $A=M \Delta M^{\top}$. Set $\tilde{\xi}=M^{\top} \xi$ and $\tilde{b}=M^{\top} b$. Then $\Delta=E\left(\tilde{\xi} \tilde{\xi}^{\top} \mid \mathcal{G}\right), \tilde{b}=E(\tilde{\xi} \eta \mid \mathcal{G}), E\left(\tilde{\xi}_{i}^{2} \mid \mathcal{G}\right)=\Delta_{i i}>0$ by hypothesis, and

$$
\begin{aligned}
b^{\top} A^{-1} b & =\tilde{b}^{\top} \Delta^{-1} \tilde{b} \\
& =\sum_{i=1}^{d} \frac{E^{2}\left(\tilde{\xi}_{i} \eta \mid \mathcal{G}\right)}{E\left(\tilde{\xi}_{i}^{2} \mid \mathcal{G}\right)} \\
& \leq d E\left(\eta^{2} \mid \mathcal{G}\right) \text { a.s. }
\end{aligned}
$$


from Cauchy-Schwarz inequality. Hence

$$
E\left\{\left(\varphi^{\top} \xi\right)^{2}\right\} \leq p E\left(\eta^{2}\right)<\infty .
$$

Next, let $\psi$ be any random vector in $\mathcal{G}$ such that $\psi^{\top} \xi \in L^{2}$. Then

$$
E\left\{\left(\psi^{\top} \xi-\eta\right)^{2}\right\}=E\left[E\left\{\left(\psi^{\top} \xi-\eta\right)^{2} \mid \mathcal{G}\right\}\right]
$$

and it is easy to check that

$$
\begin{aligned}
E\left\{\left(\psi^{\top} \xi-\eta\right)^{2} \mid \mathcal{G}\right\} & =\psi^{\top} A \psi-2 \psi^{\top} b+c \\
& =(\psi-\varphi)^{\top} A(\psi-\varphi)+\varphi^{\top} A \varphi-2 \varphi^{\top} b+c \\
& =(\psi-\varphi)^{\top} A(\psi-\varphi)+E\left\{\left(\varphi^{\top} \xi-\eta\right)^{2} \mid \mathcal{G}\right\}
\end{aligned}
$$

Hence the result.

\section{Proof of the main results}

In this section, we will prove the two main results, using the propositions proved in Appendix C.

\section{D.1 Proof of Theorem 1}

Recall that the process $\varphi=\left(\varphi_{t}\right)_{t=0}^{T}$ is predictable. For any $1 \leq t \leq T$, set $\Delta_{t}=S_{t}-$ $E\left(S_{t} \mid \mathcal{F}_{t-1}\right)$ and

$$
G_{t}=\varphi_{t}^{\top} \Delta_{t}-\left\{C_{t}-E\left(C_{t} \mid \mathcal{F}_{t-1}\right)\right\}
$$

where $C_{T}=C$ and

$$
\beta_{t-1} C_{t-1}=E\left(\beta_{t} C_{t} \mid \mathcal{F}_{t-1}\right)-\varphi_{t}^{\top} E\left(\beta_{t} S_{t}-\beta_{t-1} S_{t-1} \mid \mathcal{F}_{t-1}\right)
$$

It follows from equations (9)-(10) that

$$
\beta_{t} G_{t}=\beta_{t-1} C_{t-1}-\beta_{t} C_{t}+\varphi_{t}^{\top}\left(\beta_{t} S_{t}-\beta_{t-1} S_{t-1}\right), \quad 1 \leq t \leq T .
$$

Note that the $G_{t} \in \mathcal{F}_{t}$ and $E\left(G_{t} \mid \mathcal{F}_{t-1}\right)=0$, for all $1 \leq t \leq T$. Moreover, using (4)-(5) and (11), one gets

$$
\sum_{t=1}^{T} \beta_{t} G_{t}=C_{0}-\beta_{T} C+\sum_{t=1}^{T} \varphi_{t}^{\top}\left(\beta_{t} S_{t}-\beta_{t-1} S_{t-1}\right)=G+C_{0}-V_{0}
$$


and $E(G)=E\left(G \mid \mathcal{F}_{0}\right)=C_{0}-V_{0}$, since $E\left(G_{t} \mid \mathcal{F}_{t-1}\right)=0$ for all $t=1, \ldots, T$. It also follows from well known properties of conditional expectations that

$$
\begin{aligned}
E\left(G^{2}\right) & =E\left(G^{2} \mid \mathcal{F}_{0}\right)=\left(C_{0}-V_{0}\right)^{2}+\sum_{t=1}^{T} E\left(\beta_{t}^{2} G_{t}^{2} \mid \mathcal{F}_{0}\right) \\
& =\left(C_{0}-V_{0}\right)^{2}+\sum_{t=1}^{T} E\left\{\beta_{t}^{2} E\left(G_{t}^{2} \mid \mathcal{F}_{t-1}\right) \mid \mathcal{F}_{0}\right\}
\end{aligned}
$$

Because $G_{t}$ depends only on $\varphi_{t}, \ldots, \varphi_{T}$ through $C_{t}$, to minimize $E\left(G^{2}\right)$, it suffices to find $\varphi_{T}$ minimizing $E\left(G_{T}^{2} \mid \mathcal{F}_{0}\right)$, then to find $\varphi_{T-1}$ minimizing $E\left(G_{T-1}^{2} \mid \mathcal{F}_{0}\right)$ and so on. Doing so, we will find the minimum since each term is non negative. Having found the optimal $\varphi$, one obtains that the optimal choice for $V_{0}$ is $C_{0}$.

First, note that $G_{T}=\xi_{T}^{\top} \varphi_{T}-\eta_{T}$, where $\xi_{T}=\Delta_{T}=S_{T}-E\left(S_{T} \mid \mathcal{F}_{T-1}\right)$ and $\eta_{T}=$ $C-E\left(C \mid \mathcal{F}_{T-1}\right)=C_{T}-E\left(C_{T} \mid \mathcal{F}_{T-1}\right)$.

Using Proposition 2, one can conclude that

$$
\varphi_{T}=\left(\Sigma_{T}\right)^{-1} E\left(\xi_{T} \eta_{T} \mid \mathcal{F}_{T-1}\right)=\left(\Sigma_{T}\right)^{-1} E\left(\xi_{T} C_{T} \mid \mathcal{F}_{T-1}\right)
$$

minimizes $E\left(G_{T}^{2} \mid \mathcal{F}_{0}\right)$. Having found the optimal $\varphi_{T}$, one can define $C_{T-1}$ as in (10).

Suppose now that $\varphi_{T}, \ldots, \varphi_{t}$ have been defined and define $G_{t-1}$ and $C_{t-1}$ according to (9) and (10). Then one can use again Proposition (2) to conclude $\varphi_{t-1}$ given by (6) minimizes $E\left(G_{t-1}^{2} \mid \mathcal{F}_{0}\right)$.

Therefore the risk $E\left(G^{2} \mid \mathcal{F}_{0}\right)$ is minimized by choosing the $\varphi_{t}$ 's according to (6). Finally, using (12), the optimal value of $V_{0}$ is $C_{0}$. This completes the proof.

\section{D.2 Proof of Corollary 1}

The proof of the representation $C_{t-1}=E\left(C_{t} U_{t} \mid \mathcal{F}_{t-1}\right)$ follows directly from Theorem 1 . In fact, using equations (6) and (7), one obtains

$$
\begin{aligned}
\beta_{t-1} C_{t-1}= & E\left(\beta_{t} C_{t} \mid \mathcal{F}_{t-1}\right)-\varphi_{t}^{\top} E\left(\beta_{t} S_{t}-\beta_{t-1} S_{t-1} \mid \mathcal{F}_{t-1}\right) \\
= & E\left(\beta_{t} C_{t} \mid \mathcal{F}_{t-1}\right) \\
& -E\left\{C_{t} \Delta_{t}^{\top}\left(\Sigma_{t}\right)^{-1} E\left(\beta_{t} S_{t}-\beta_{t-1} S_{t-1} \mid \mathcal{F}_{t-1}\right) \mid \mathcal{F}_{t-1}\right\} \\
= & E\left(C_{t} U_{t} \mid \mathcal{F}_{t-1}\right),
\end{aligned}
$$

where $U_{t}$ is defined by (8). One can easily see that $E\left(U_{t} \mid \mathcal{F}_{t-1}\right)=1$, so $\left(M_{t}\right)_{t=0}^{T}$ is a martingale.

It only remains to prove that $\beta_{t} S_{t} M_{t}$ is a martingale. All is needed is to prove that $E\left(\beta_{t} S_{t} U_{t} \mid \mathcal{F}_{t-1}\right)=\beta_{t-1} S_{t-1}$. To this end, let $t \in\{1, \ldots, T\}$ be given and set $\xi_{t}=$ 
$E\left(\beta_{t} S_{t}-\beta_{t-1} S_{t-1} \mid \mathcal{F}_{t-1}\right)$. Note that

$$
\beta_{t} S_{t} U_{t}=\beta_{t} S_{t}-\left\{\Delta_{t}+E\left(S_{t} \mid \mathcal{F}_{t-1}\right)\right\} \Delta_{t}^{\top}\left(\Sigma_{t}\right)^{-1} \xi_{t} .
$$

Next, since $E\left(\Delta_{t} \mid \mathcal{F}_{t-1}\right)=0$, one has

$$
\begin{aligned}
E\left(\beta_{t} S_{t} U_{t} \mid \mathcal{F}_{t-1}\right)= & E\left(\beta_{t} S_{t} \mid \mathcal{F}_{t-1}\right)-E\left(\Delta_{t} \Delta_{t}^{\top} \mid \mathcal{F}_{t-1}\right)\left(\Sigma_{t}\right)^{-1} \xi_{t} \\
& -E\left(S_{t} \mid \mathcal{F}_{t-1}\right) E\left(\Delta_{t}^{\top} \mid \mathcal{F}_{t-1}\right)\left(\Sigma_{t}\right)^{-1} \xi_{t} \\
= & E\left(\beta_{t} S_{t} \mid \mathcal{F}_{t-1}\right)-\Sigma_{t}\left(\Sigma_{t}\right)^{-1} \xi_{t}-0 \\
= & E\left(\beta_{t} S_{t} \mid \mathcal{F}_{t-1}\right)-\xi_{t}=\beta_{t-1} S_{t-1}
\end{aligned}
$$

Hence the result. 